\title{
General methods of convergence and summability
}

\author{
Francisco Javier García-Pacheco ${ }^{1}$, Ramazan Kama² and María del Carmen Listán-García ${ }^{1 *}$
}

\author{
${ }^{*}$ Correspondence: \\ mariadelcarmen.listan@uca.es \\ 'Department of Mathematics, \\ University of Cadiz, Puerto Real \\ 11510, Spain \\ Full list of author information is \\ available at the end of the article
}

\begin{abstract}
This paper is on general methods of convergence and summability. We first present the general method of convergence described by free filters of $\mathbb{N}$ and study the space of convergence associated with the filter. We notice that $c(X)$ is always a space of convergence associated with a filter (the Frechet filter); that if $X$ is finite dimensional, then $\ell_{\infty}(X)$ is a space of convergence associated with any free ultrafilter of $\mathbb{N}$; and that if $X$ is not complete, then $\ell_{\infty}(X)$ is never the space of convergence associated with any free filter of $\mathbb{N}$. Afterwards, we define a new general method of convergence inspired by the Banach limit convergence, that is, described through operators of norm 1 which are an extension of the limit operator. We prove that $\ell_{\infty}(X)$ is always a space of convergence through a certain class of such operators; that if $X$ is reflexive and 1-injective, then $c(X)$ is a space of convergence through a certain class of such operators; and that if $X$ is not complete, then $c(X)$ is never the space of convergence through any class of such operators. In the meantime, we study the geometric structure of the set $\mathcal{H B}(\lim ):=\left\{T \in \mathcal{B}\left(\ell_{\infty}(X), X\right):\left.T\right|_{c(X)}=\lim\right.$ and $\left.\|T\|=1\right\}$ and prove that $\mathcal{H} \mathcal{B}(\lim )$ is a face of $B_{\mathcal{L}_{X}^{0}}$ if $X$ has the Bade property, where $\mathcal{L}_{X}^{0}:=\left\{T \in \mathcal{B}\left(\ell_{\infty}(X), X\right)\right.$ : $\left.c_{0}(X) \subseteq \operatorname{ker}(T)\right\}$. Finally, we study the multipliers associated with series for the above methods of convergence.
\end{abstract}

MSC: 47A05

Keywords: Methods; Convergence; Summability

\section{Introduction}

Recall that if $f: D \rightarrow X$ is a function from a set $D$, endowed with a filter base $B$, into a topological space $X$, then

$$
\begin{aligned}
\lim _{B} f & :=\lim f(B) \\
& =\left\{x \in X: \mathcal{N}_{x} \subseteq \mathcal{J}(f(B))\right\} \\
& =\left\{x \in X: \forall U \in \mathcal{N}_{x} \exists A \in B \text { such that } f(A) \subseteq U\right\} \\
& =\left\{x \in X: \forall U \in \mathcal{N}_{x} \exists A \in B \text { such that } A \subseteq f^{-1}(U)\right\} \\
& =\left\{x \in X: \forall U \in \mathcal{N}_{x} f^{-1}(U) \in \mathcal{J}(B)\right\},
\end{aligned}
$$

(c) The Author(s) 2021. This article is licensed under a Creative Commons Attribution 4.0 International License, which permits use, sharing, adaptation, distribution and reproduction in any medium or format, as long as you give appropriate credit to the original author(s) and the source, provide a link to the Creative Commons licence, and indicate if changes were made. The images or other third party material in this article are included in the article's Creative Commons licence, unless indicated otherwise in a credit line to the material. If material is not included in the article's Creative Commons licence and your intended use is not permitted by statutory regulation or exceeds the permitted use, you will need to obtain permission directly from the copyright holder. To view a copy of this licence, visit http://creativecommons.org/licenses/by/4.0/. 
where $\mathcal{N}_{x}$ is the filter of neighborhoods of $x, \mathcal{J}(f(B))$ is the filter of $X$ generated by the filter base $f(B)$, and $\mathcal{J}(B)$ is the filter generated by the filter base $B$. Notice that if $B_{1}$ and $B_{2}$ are filter bases and $B_{1} \subseteq B_{2}$, then $f\left(B_{1}\right) \subseteq f\left(B_{2}\right)$ and $\mathcal{J}\left(f\left(B_{1}\right)\right) \subseteq \mathcal{J}\left(f\left(B_{2}\right)\right)$, and hence

$$
\lim _{B_{1}} f=\lim f\left(B_{1}\right) \subseteq \lim f\left(B_{2}\right)=\lim _{B_{2}} f .
$$

The usual convergence of nets is in fact defined in the previous way. Indeed, if $f$ is a net, then $D$ is a directed set and $B$ is the filter base associated with $D$, that is, $B:=\{\uparrow d: d \in D\}$.

The convergence given by Equation (1.1) can also be expressed in terms of ideals. Recall that an ideal is the dual concept of a filter. As a matter of fact, if $F$ is a filter (base) in a nonempty set $D$, then $I_{F}:=\{D \backslash A: A \in F\}$ is an ideal (base) on $D$, and conversely, if $I$ is an ideal (base) of $D$, then $F_{I}:=\{D \backslash A: A \in I\}$ is a filter (base) in $D$. In this sense, the limit through an ideal base $B$ is defined as the limit through the associated filter base $F_{B}$. In other words, if $B$ is an ideal base in $D$, then

$$
\begin{aligned}
\lim _{B} f & :=\lim _{F_{B}} f \\
& =\lim f\left(F_{B}\right) \\
& =\left\{x \in X: \mathcal{N}_{x} \subseteq \mathcal{J}\left(f\left(F_{B}\right)\right)\right\} \\
& =\left\{x \in X: \forall U \in \mathcal{N}_{x} \exists A \in B \text { such that } f(D \backslash A) \subseteq U\right\} \\
& =\left\{x \in X: \forall U \in \mathcal{N}_{x} \exists A \in B \text { such that } D \backslash A \subseteq f^{-1}(U)\right\} \\
& =\left\{x \in X: \forall U \in \mathcal{N}_{x} \exists A \in B \text { such that } D \backslash f^{-1}(U) \subseteq A\right\} \\
& =\left\{x \in X: \forall U \in \mathcal{N}_{x} \exists A \in B \text { such that } f^{-1}(X \backslash U) \subseteq A\right\} \\
& =\left\{x \in X: \forall U \in \mathcal{N}_{x} f^{-1}(X \backslash U) \in \mathcal{I}(B)\right\},
\end{aligned}
$$

where as expected $\mathcal{I}(B)$ is the ideal generated by the ideal base $B$.

In the case of sequences we obtain the usual notions of convergence. Indeed, in this case $D=\mathbb{N}$, and if $B$ is a filter base in $\mathbb{N}$, then

$$
\lim _{B}\left(x_{n}\right)=\left\{x \in X: \forall U \in \mathcal{N}_{x}\left\{n \in \mathbb{N}: x_{n} \in U\right\} \in \mathcal{J}(B)\right\},
$$

and if $B$ is an ideal base in $\mathbb{N}$, then

$$
\lim _{B}\left(x_{n}\right)=\left\{x \in X: \forall U \in \mathcal{N}_{x}\left\{n \in \mathbb{N}: x_{n} \notin U\right\} \in \mathcal{I}(B)\right\} .
$$

If $X$ is a metric space, then the previous sets can be rewritten as

$$
x=\lim _{B}\left(x_{n}\right) \quad \Leftrightarrow \quad \forall \varepsilon>0 \quad\left\{n \in \mathbb{N}: d\left(x_{n}, x\right)<\varepsilon\right\} \in \mathcal{J}(B)
$$

for $B$ a filter base and

$$
x=\lim _{B}\left(x_{n}\right) \quad \Leftrightarrow \quad \forall \varepsilon>0 \quad\left\{n \in \mathbb{N}: d\left(x_{n}, x\right) \geq \varepsilon\right\} \in \mathcal{I}(B)
$$

for $B$ an ideal base. Finally, notice that the Frechet filter of $\mathbb{N}, \mathcal{F}_{\mathbb{N}}:=\left\{A \subseteq \mathbb{N}: \mathbb{N} \backslash A \in \phi_{0}(\mathbb{N})\right\}$, is precisely the filter of reduced neighborhoods of $\infty$ in the one-point compactification of 
$\mathbb{N}, \overline{\mathbb{N}}:=\mathbb{N} \cup\{\infty\}$, where $\phi_{0}(\mathbb{N})$ is the family of finite subsets of $\mathbb{N}$. In other words, $\mathcal{N}_{\infty}^{\times}(\overline{\mathbb{N}})=$ $\mathcal{F}_{\mathbb{N}}$. This means that the usual convergence of sequences coincides with the convergence through the Frechet filter of $\mathbb{N}$. On the other hand, it is clear that $F_{\phi_{0}(\mathbb{N})}=\mathcal{F}_{\mathbb{N}}$ and $I_{\mathcal{F}_{\mathbb{N}}}=$ $\phi_{0}(\mathbb{N})$. As a consequence, if $F$ is a filter of $\mathbb{N}$ containing the Frechet filter or $I$ is an ideal of $\mathbb{N}$ containing $\phi_{0}(\mathbb{N})$, then the usual convergence of a sequence implies the convergence of that sequence through $F$ or $I$, respectively.

The uniform convergence of matrices or sequences is described as follows: if $\mathcal{A}:=$ $\left(a_{i j}\right)_{i, j \in I \times J}$ is a matrix of index sets $I$ and $J$ in a topological space $X$ and $\mathcal{B}$ is a filter base in $J$, then

$$
\mathrm{u} \lim _{\mathcal{B}}\left(a_{i j}\right):=\left\{x \in X: \forall U \in \mathcal{N}_{x} \exists B \in \mathcal{B} \forall i \in I B \subseteq\left\{j \in J: a_{i j} \in U\right\}\right\}
$$

Observe that $\mathrm{u} \lim _{\mathcal{B}}\left(a_{i j}\right) \subseteq \lim _{\mathcal{B}}\left(a_{i j}\right)$ for all $i \in I$.

If $X$ is a topological vector space, then an $X$-sequence space is simply a vector subspace $\mathcal{V}$ of $X^{\mathbb{N}}$ endowed with a vector topology for which the coordinate maps $\delta_{n}: \mathcal{V} \rightarrow X$ are continuous; in other words, the vector topology of $\mathcal{V}$ is finer than the initial topology $\sigma\left(\mathcal{V},\left\{\delta_{n}: n \in \mathbb{N}\right\}\right)$ of $\mathcal{V}$ generated by $\left\{\delta_{n}: n \in \mathbb{N}\right\}$. Keep in mind that this initial topology is precisely the inherited topology from the product topology on $X^{\mathbb{N}}$. Notice that if $X$ is Hausdorff or locally convex, then $X^{\mathbb{N}}$ is Hausdorff or locally convex, respectively, therefore $\sigma\left(\mathcal{V},\left\{\delta_{n}: n \in \mathbb{N}\right\}\right)$ is also Hausdorff or locally convex, respectively, and thus $\mathcal{V}$ is Hausdorff or locally convex, respectively.

If $\left(X_{i}\right)_{i \in I}$ is a family of topological vector spaces, then

$$
\left\{\prod_{i \in I} U_{i}: U_{i} \in \mathcal{N}_{0}\left(X_{i}\right)\right\}
$$

is basis of zero neighborhoods for a vector topology on $\prod_{i \in I} X_{i}$ called the uniform convergence topology. This topology is clearly finer than the product topology, which is precisely the pointwise convergence topology. If $X$ is a topological vector space and $\mathcal{V}$ is an $X$-sequence space, then the uniform convergence topology on $\mathcal{V}$ is the inherited topology on $\mathcal{V}$ from the uniform convergence topology of $X^{\mathbb{N}}$. If $X$ is a normed space, then the sup norm on $\ell_{\infty}(X)$ precisely induces the uniform convergence topology.

A subset $A$ of a topological vector space $X$ is said to be bounded provided that, for every zero neighborhood $V$ of $X$, there exists $\alpha \in \mathbb{K}$ such that $A \subseteq \alpha V$. Note that $\ell_{\infty}(X)$ and $c(X)$ stand for the vector space of bounded sequences on $X$ and for the vector space of convergent sequences on $X$, respectively. It is clear that every convergent sequence in a Hausdorff topological vector space is bounded (due to the existence of a fundamental system of balanced and absorbing neighborhoods of zero), therefore $c(X) \subseteq \ell_{\infty}(X)$.

Throughout this manuscript we rely on the categorical concept of injective object. We recall this concept in the category of Banach spaces.

Let $X$ be a Banach space and $k \geq 1$. A subspace $Y$ is said to be $k$-complemented in $X$ if there exists a projection $P: X \rightarrow Y$ such that $\|P\| \leq k$. We also say that $P$ is a $k$-projection.

Let $k \geq 1$. A Banach space $X$ is called $k$-injective if it is $k$-complemented in every $Y$ such that $X \subseteq Y$.

In accordance with [9, p. 123], a Banach space $X$ is $k$-injective if and only if it satisfies any of the following (for all arbitrary Banach spaces $Y, Z$ ): 
- If $X \subseteq Y$ and $T: X \rightarrow Z$ is linear and continuous, then there exists a continuous linear extension $S: Y \rightarrow Z$ with $\|S\| \leq k\|T\|$.

- If $Z \subseteq Y$ and $T: Z \rightarrow X$ is linear and continuous, then there exists a continuous linear extension $S: Y \rightarrow X$ with $\|S\| \leq k\|T\|$.

- If $T: X \rightarrow Y$ is a linear isometry, there exists a continuous linear extension $S: Y \rightarrow X$ such that $\|S\| \leq k$ and $S \circ T$ is the identity.

The 1-injective spaces are exactly the $\mathcal{C}(K)$ spaces for $K$ extremely disconnected, that is, the closure of every open set in $K$ is open (see [17]). As a consequence, the reflexive 1-injective Banach spaces are precisely the spaces $\ell_{\infty}^{n}$ for $n \in \mathbb{N}$.

\section{Filter convergence}

\subsection{Simple filter convergence}

In this subsection we define the space of convergence associated with a free filter of $\mathbb{N}$ and prove several properties verified by it.

Definition 2.1 Let $\mathcal{F}$ be a free filter of $\mathbb{N}$ or, equivalently, a filter of $\mathbb{N}$ containing the Frechet filter. Let $X$ be a normed space. We define the space of $\mathcal{F}$-convergence as

$$
c_{\mathcal{F}}(X):=\left\{\left(x_{n}\right)_{n \in \mathbb{N}} \in \ell_{\infty}(X): \exists \lim _{\mathcal{F}} x_{n}\right\} .
$$

The $\mathcal{F}$-limit operator is defined as

$$
\begin{aligned}
\mathcal{F} \lim : & c_{\mathcal{F}}(X) \rightarrow X, \\
& \left(x_{n}\right)_{n \in \mathbb{N}} \mapsto \mathcal{F} \lim x_{n}:=\lim _{\mathcal{F}} x_{n} .
\end{aligned}
$$

As nontrivial examples of free filters of $\mathbb{N}$, we can consider the sets with natural density 1 , which yield the so-called statistical convergence $[11,20]$. Recently, a generalization of the concept of density was given by means of a modulus function $f$; in this case, a free filter can be obtained by using the complements of sets with null $f$-density $[4,5]$.

For the upcoming lemma, it is important to bear in mind the following remark.

Remark 2.2 If $\mathcal{F}$ is an ultrafilter in a set $A$ and $f: A \rightarrow B$ is a map, then $\mathcal{J}(f(\mathcal{F}))$ is an ultrafilter in $B$.

Theorem 2.3 Let $\mathcal{F}$ be a free filter of $\mathbb{N}$. Let $X$ be a normed space. Then:

(1) $c_{\mathcal{F}}(X)$ is a subspace of $\ell_{\infty}(X)$ containing $c(X)$ and $\mathcal{F} \lim$ is linear.

(2) If $\mathcal{G}$ is another filter of $\mathbb{N}$ containing $\mathcal{F}$, then $c_{\mathcal{F}}(X) \subseteq c_{\mathcal{G}}(X)$ and $\left.\mathcal{G} \lim \right|_{\mathcal{F}_{\mathcal{F}}(X)}=\mathcal{F}$ lim.

(3) $c(X)=c_{\mathcal{F}_{\mathbb{N}}}(X)$ and $\mathcal{F}_{\mathbb{N}} \lim =\lim$.

(4) $\|\mathcal{F} \lim \|=1$.

(5) If $\mathcal{F}$ is a free ultrafilter and $X$ is finite dimensional, then $c_{\mathcal{F}}(X)=\ell_{\infty}(X)$.

Proof

(1) Let $x:=\lim _{\mathcal{F}} x_{n}$ and $y:=\lim _{\mathcal{F}} y_{n}$. Let $U$ be an open neighborhood of $x+y$. There are open neighborhoods $V_{x}$ and $V_{y}$ of $x$ and $y$, respectively, such that $V_{x}+V_{y} \subseteq U$. Note that $\left\{n \in \mathbb{N}: x_{n} \in V_{x}\right\} \in \mathcal{F}$ and $\left\{n \in \mathbb{N}: y_{n} \in V_{y}\right\} \in \mathcal{F}$. In particular,

$$
\left\{n \in \mathbb{N}: x_{n} \in V_{x} \text { and } y_{n} \in V_{y}\right\}=\left\{n \in \mathbb{N}: x_{n} \in V_{x}\right\} \cap\left\{n \in \mathbb{N}: y_{n} \in V_{y}\right\} \in \mathcal{F} \text {. }
$$


On the other hand,

$$
\left\{n \in \mathbb{N}: x_{n} \in V_{x} \text { and } y_{n} \in V_{y}\right\} \subseteq\left\{n \in \mathbb{N}: x_{n}+y_{n} \in U\right\},
$$

which implies that $\left\{n \in \mathbb{N}: x_{n}+y_{n} \in U\right\} \in \mathcal{F}$. This shows that $x+y \in \lim _{\mathcal{F}}\left(x_{n}+y_{n}\right)$. Since $X$ is Hausdorff, we have that $x+y=\lim _{\mathcal{F}}\left(x_{n}+y_{n}\right)$. Now let $\lambda \in \mathbb{K}$. Let $U$ be an open neighborhood of $\lambda x$. There is an open neighborhood $V$ of $x$ such that $\lambda V \subseteq U$. Observe that $\left\{n \in \mathbb{N}: x_{n} \in V\right\} \in \mathcal{F}$. On the other hand,

$$
\left\{n \in \mathbb{N}: x_{n} \in V\right\} \subseteq\left\{n \in \mathbb{N}: \lambda x_{n} \in \lambda V\right\} \subseteq\left\{n \in \mathbb{N}: \lambda x_{n} \in U\right\},
$$

which implies $\left\{n \in \mathbb{N}: \lambda x_{n} \in U\right\} \in \mathcal{F}$. This shows that $\lambda x \in \lim _{\mathcal{F}}\left(\lambda x_{n}\right)$. Since $X$ is Hausdorff, we have that $\lambda x=\lim _{\mathcal{F}}\left(\lambda x_{n}\right)$. Finally, let us prove that $c(X) \subseteq c_{\mathcal{F}}(X)$. Let $\left(x_{n}\right)_{n \in \mathbb{N}}$ be a convergent sequence to $x \in X$. We will show that $\lim _{\mathcal{F}} x_{n}=x$. Let $\mathcal{F}_{\mathbb{N}}$ denote the Frechet filter of $\mathbb{N}$. Since $\mathcal{F}_{\mathbb{N}} \subseteq \mathcal{F}$, we know that

$$
\lim _{n \rightarrow \infty} x_{n}=\lim _{\mathcal{F}_{\mathbb{N}}} x_{n} \subseteq \lim _{\mathcal{F}} x_{n}
$$

Since $X$ is Hausdorff, $x=\lim _{\mathcal{F}} x_{n}$.

(2) Simply observe that since $\mathcal{F} \subseteq \mathcal{G}$, we have that $\lim _{\mathcal{F}} x_{n} \subseteq \lim _{\mathcal{G}} x_{n}$, which implies that $c_{\mathcal{F}}(X) \subseteq c_{\mathcal{G}}(X)$. The Hausdorff character of $X$ implies that $\left.\mathcal{G} \lim \right|_{c_{\mathcal{F}}(X)}=\mathcal{F} \lim$.

(3) By (1) we have that $c(X) \subseteq c_{\mathcal{F}_{\mathbb{N}}}(X)$. Let $\left(x_{n}\right)_{n \in \mathbb{N}} \in c_{\mathcal{F}_{\mathbb{N}}}(X)$. We know that $\lim _{\mathcal{F}_{\mathbb{N}}} x_{n}=\lim _{n \rightarrow \infty} x_{n}$, which means that $\left(x_{n}\right)_{n \in \mathbb{N}} \in c(X)$ and $\mathcal{F}_{\mathbb{N}} \lim =\lim$.

(4) Since $\left.\mathcal{F} \lim \right|_{c(X)}=\left.\mathcal{F} \lim \right|_{\mathcal{F}_{\mathbb{N}}(X)}=\mathcal{F}_{\mathbb{N}} \lim =\lim$ and $\|\lim \|=1$, we conclude that $\|\mathcal{F} \lim \| \geq 1$. Now, let $\left(x_{n}\right)_{n \in \mathbb{N}} \in \mathrm{B}_{\mathcal{F}_{\mathcal{F}}(X)}$. Let $x:=\lim _{\mathcal{F}} x_{n}$. Suppose that $\|x\|>1$. Let $V$ be an open neighborhood of $x$ such that $V \cap \mathrm{B}_{X}=\varnothing$. Notice that

$\left\{n \in \mathbb{N}: x_{n} \in V\right\} \in \mathcal{F}$. However, $\left\{n \in \mathbb{N}: x_{n} \in V\right\}=\varnothing$ since $\left\|x_{n}\right\| \leq 1$. This implies the contradiction that $\varnothing \in \mathcal{F}$.

(5) Let $\left(x_{n}\right)_{n \in \mathbb{N}} \in \ell_{\infty}(X)$. Note that $\mathrm{B}_{X}\left(0,\left\|\left(x_{n}\right)_{n \in \mathbb{N}}\right\|_{\infty}\right)$ is compact and $\mathcal{G}:=\left\{\left\{x_{n}: n \in F\right\}: F \in \mathcal{F}\right\}$ is a filter base in $\mathrm{B}_{X}\left(0,\left\|\left(x_{n}\right)_{n \in \mathbb{N}}\right\|_{\infty}\right)$ whose induced filter is an ultrafilter in view of Remark 2.2. The compactness of $\mathrm{B}_{X}\left(0,\left\|\left(x_{n}\right)_{n \in \mathbb{N}}\right\|_{\infty}\right)$ allows that $\lim (\mathcal{G})=\lim (\mathcal{J}(\mathcal{G})) \neq \varnothing$. Finally, notice that $\lim _{\mathcal{F}} x_{n}=\lim (\mathcal{G})$.

Theorem 2.4 Let $X$ be a noncomplete normed space. No free filter $\mathcal{F}$ of $\mathbb{N}$ verifies that $c_{\mathcal{F}}(X)=\ell_{\infty}(X)$.

Proof Fix a nonconvergent Cauchy sequence $\left(x_{n}\right)_{n \in \mathbb{N}}$ of $X$. Assume to the contrary that there exists $x:=\lim _{\mathcal{F}} x_{n}$. We will reach the contradiction that $\lim _{n \rightarrow \infty} x_{n}=x$. Let $\varepsilon>0$. Fix $n_{1} \in \mathbb{N}$ such that $\left\|x_{n}-x_{m}\right\|<\frac{\varepsilon}{2}$ for all $n, m \geq n_{1}$. Note that $\left\{n \in \mathbb{N}:\left\|x_{n}-x\right\|<\frac{\varepsilon}{2}\right\} \in \mathcal{F}$. Furthermore, the previous set has to be infinite because $\mathcal{F}$ contains the Frechet filter of $\mathbb{N}$. Let $n_{2}:=\min \left\{n \in \mathbb{N}:\left\|x_{n}-x\right\|<\frac{\varepsilon}{2}\right\}$. Take $n_{0}:=\max \left\{n_{1}, n_{2}\right\}$. If $n \geq n_{0}$, then we have two possibilities:

- $\left\|x_{n}-x\right\|<\frac{\varepsilon}{2}<\varepsilon$.

- Take $m>n$ sufficiently large such that $\left\|x_{m}-x\right\|<\frac{\varepsilon}{2}<\varepsilon$. Then $\left\|x_{n}-x\right\| \leq\left\|x_{n}-x_{m}\right\|+$ $\left\|x_{m}-x\right\|<\varepsilon$.

This shows that $\lim _{n \rightarrow \infty} x_{n}=x$. 
The last result in this subsection shows the existence of unbounded sequences that are $\mathcal{F}$-convergent.

Theorem 2.5 Let $X$ be any nonzero normed space. For every free filter $\mathcal{F}$ of $\mathbb{N}$ containing strictly the Frechet filter of $\mathbb{N}$, there exists an unbounded sequence $\left(x_{n}\right)_{n \in \mathbb{N}}$ such that $\left(x_{n}\right)_{n \in \mathbb{N}}$ is $\mathcal{F}$-convergent to 0 .

Proof Since $\mathcal{F}$ contains strictly the Frechet filter of $\mathbb{N}$, there exists $A \in \mathcal{F}$ infinite whose complementary $\mathbb{N} \backslash A$ is also infinite. Fix $x \in X \backslash\{0\}$ and define the sequence

$$
x_{n}:= \begin{cases}0 & \text { if } n \in A, \\ n x & \text { if } n \in \mathbb{N} \backslash A .\end{cases}
$$

Observe that $\left(x_{n}\right)_{n \in \mathbb{N}}$ is unbounded. Now observe that if $U$ is any neighborhood of 0 , then $\left\{n \in \mathbb{N}: x_{n} \in U\right\} \supseteq A$ and $A \in \mathcal{F}$, which implies that $\left\{n \in \mathbb{N}: x_{n} \in U\right\} \in \mathcal{F}$. This shows that $\lim _{\mathcal{F}} x_{n}=0$.

\subsection{Composed filter convergence}

The following result is a generalization of [7, Theorem 1].

Theorem 2.6 Let $X$ be a Banach space. Let $\mathcal{F}$ be a free ultrafilter in $\mathbb{N}$. Let $T: \ell_{\infty}\left(X^{*}\right) \rightarrow$ $\ell_{\infty}\left(X^{*}\right)$ be a linear and continuous operator. The operator

$$
\begin{aligned}
T_{\mathcal{F}}: & \ell_{\infty}\left(X^{*}\right) \rightarrow X^{*}, \\
& \left(x_{n}^{*}\right)_{n \in \mathbb{N}} \mapsto w^{*} \lim _{\mathcal{F}} T\left(\left(x_{n}^{*}\right)_{n \in \mathbb{N}}\right)
\end{aligned}
$$

verifies the following properties:

(1) $T_{\mathcal{F}}$ is linear and continuous and $\left\|T_{\mathcal{F}}\right\| \leq\|T\|$.

(2) If $T\left(c\left(X^{*}\right)\right) \subseteq c\left(X^{*}\right)$, then $\left.T_{\mathcal{F}}\right|_{c\left(X^{*}\right)}=\lim _{n \rightarrow \infty} T\left(\left(x_{n}^{*}\right)_{n \in \mathbb{N}}\right)$.

Proof

(1) Denote $\left(y_{n}^{*}\right)_{n \in \mathbb{N}}:=T\left(\left(x_{n}^{*}\right)_{n \in \mathbb{N}}\right)$. Note that $\mathrm{B}_{X^{*}}\left(0,\left\|T\left(\left(x_{n}^{*}\right)_{n \in \mathbb{N}}\right)\right\|_{\infty}\right)$ is $w^{*}$-compact and

$$
\mathcal{G}:=\left\{\left\{\left(y_{n}^{*}\right): n \in F\right\}: F \in \mathcal{F}\right\}
$$

is an ultrafilter in $\mathrm{B}_{X^{*}}\left(0,\left\|T\left(\left(x_{n}^{*}\right)_{n \in \mathbb{N}}\right)\right\|_{\infty}\right)$ in view of Remark 2.2. The $w^{*}$-compactness of $\mathrm{B}_{X^{*}}\left(0,\left\|T\left(\left(x_{n}^{*}\right)_{n \in \mathbb{N}}\right)\right\|_{\infty}\right)$ allows that $w^{*} \lim (\mathcal{G}) \neq \varnothing$. Observe that $w^{*} \lim _{\mathcal{F}} T\left(\left(x_{n}^{*}\right)_{n \in \mathbb{N}}\right)=w^{*} \lim (\mathcal{G})$. This shows that $T_{\mathcal{F}}$ is well defined. Let us show now that $T_{\mathcal{F}}$ is linear. Let $x^{*}:=w^{*} \lim _{\mathcal{F}} T\left(\left(x_{n}^{*}\right)_{n \in \mathbb{N}}\right)$ and $y^{*}:=w^{*} \lim _{\mathcal{F}} T\left(\left(y_{n}^{*}\right)_{n \in \mathbb{N}}\right)$. Denote $\left(a_{n}^{*}\right)_{n \in \mathbb{N}}:=T\left(\left(x_{n}^{*}\right)_{n \in \mathbb{N}}\right)$ and $\left(b_{n}^{*}\right)_{n \in \mathbb{N}}:=T\left(\left(x_{n}^{*}\right)_{n \in \mathbb{N}}\right)$. Observe that $\left(a_{n}^{*}+b_{n}^{*}\right)_{n \in \mathbb{N}}=$ $\left(a_{n}^{*}\right)_{n \in \mathbb{N}}+\left(b_{n}^{*}\right)_{n \in \mathbb{N}}=T\left(\left(x_{n}^{*}\right)_{n \in \mathbb{N}}\right)+T\left(\left(y_{n}^{*}\right)_{n \in \mathbb{N}}\right)=T\left(\left(x_{n}^{*}\right)_{n \in \mathbb{N}}+\left(y_{n}^{*}\right)_{n \in \mathbb{N}}\right)=T\left(\left(x_{n}^{*}+y_{n}^{*}\right)_{n \in \mathbb{N}}\right)$. Let $U$ be a $w^{*}$-open neighborhood of $x^{*}+y^{*}$. There are $w^{*}$-open neighborhoods $V_{x^{*}}$ and $V_{y^{*}}$ of $x^{*}$ and $y^{*}$, respectively, such that $V_{x^{*}}+V_{y^{*}} \subseteq U$. Note that $\left\{n \in \mathbb{N}: a_{n}^{*} \in V_{x^{*}}\right\} \in \mathcal{F}$ and $\left\{n \in \mathbb{N}: b_{n}^{*} \in V_{y^{*}}\right\} \in \mathcal{F}$. In particular,

$$
\left\{n \in \mathbb{N}: a_{n}^{*} \in V_{x^{*}} \text { and } b_{n}^{*} \in V_{y^{*}}\right\}=\left\{n \in \mathbb{N}: a_{n}^{*} \in V_{x^{*}}\right\} \cap\left\{n \in \mathbb{N}: b_{n}^{*} \in V_{y^{*}}\right\} \in \mathcal{F} \text {. }
$$


On the other hand,

$$
\left\{n \in \mathbb{N}: a_{n}^{*} \in V_{x^{*}} \text { and } b_{n}^{*} \in V_{y^{*}}\right\} \subseteq\left\{n \in \mathbb{N}: a_{n}^{*}+b_{n}^{*} \in U\right\} \text {, }
$$

which implies that $\left\{n \in \mathbb{N}: a_{n}^{*}+b_{n}^{*} \in U\right\} \in \mathcal{F}$. This shows that

$$
x^{*}+y^{*} \in w^{*} \lim _{\mathcal{F}} T\left(\left(x_{n}^{*}\right)_{n \in \mathbb{N}}+\left(y_{n}^{*}\right)_{n \in \mathbb{N}}\right) .
$$

Since the $w^{*}$-topology on $X^{*}$ is Hausdorff, we have that $x^{*}+y^{*}=w^{*} \lim _{\mathcal{F}} T\left(\left(x_{n}^{*}+y_{n}^{*}\right)_{n \in \mathbb{N}}\right)$. Now let $\lambda \in \mathbb{K}$. Let $U$ be a $w^{*}$-open neighborhood of $\lambda x^{*}$. There is a $w^{*}$-open neighborhood $V$ of $x^{*}$ such that $\lambda V \subseteq U$. Observe that $\left\{n \in \mathbb{N}: a_{n}^{*} \in V\right\} \in \mathcal{F}$. On the other hand,

$$
\left\{n \in \mathbb{N}: a_{n}^{*} \in V\right\} \subseteq\left\{n \in \mathbb{N}: \lambda a_{n}^{*} \in \lambda V\right\} \subseteq\left\{n \in \mathbb{N}: \lambda a_{n}^{*} \in U\right\}
$$

which implies $\left\{n \in \mathbb{N}: \lambda a_{n}^{*} \in U\right\} \in \mathcal{F}$. This shows that

$$
\lambda x^{*} \in w^{*} \lim _{\mathcal{F}}\left(\lambda a_{n}^{*}\right)=w^{*} \lim _{\mathcal{F}} T\left(\lambda\left(x_{n}^{*}\right)_{n \in \mathbb{N}}\right) .
$$

Since the $w^{*}$-topology on $X^{*}$ is Hausdorff, $\lambda x^{*}=w^{*} \lim _{\mathcal{F}}\left(\lambda a_{n}^{*}\right)=w^{*} \lim _{\mathcal{F}} T\left(\lambda\left(x_{n}^{*}\right)_{n \in \mathbb{N}}\right)$. Finally, let us show that $\left\|T_{\mathcal{F}}\right\| \leq\|T\|$. In the first place, notice that if $\left(x_{n}^{*}\right)_{n \in \mathbb{N}} \in \mathrm{B}_{\ell_{\infty}\left(X^{*}\right)}$, then $T\left(\left(x_{n}^{*}\right)_{n \in \mathbb{N}}\right) \in \mathrm{B}_{\ell_{\infty}\left(X^{*}\right)}\left(0,\left\|T\left(\left(x_{n}^{*}\right)_{n \in \mathbb{N}}\right)\right\|_{\infty}\right) \subseteq \mathrm{B}_{\ell_{\infty}\left(X^{*}\right)}(0,\|T\|)$. Now if we denote $\left(a_{n}^{*}\right)_{n \in \mathbb{N}}:=T\left(\left(x_{n}^{*}\right)_{n \in \mathbb{N}}\right)$, then

$$
\left\|a_{n}^{*}\right\| \leq\left\|\left(a_{n}^{*}\right)_{n \in \mathbb{N}}\right\|_{\infty} \leq\|T\|
$$

for every $n \in \mathbb{N}$. This implies that $w^{*} \lim _{\mathcal{F}} T\left(\left(x_{n}^{*}\right)_{n \in \mathbb{N}}\right) \in \mathrm{B}_{X^{*}}(0,\|T\|)$ and hence $\left\|T_{\mathcal{F}}\right\| \leq\|T\|$.

(2) If $\left(x_{n}^{*}\right)_{n \in \mathbb{N}}$ is convergent to some $x^{*} \in X^{*}$, then $T\left(\left(x_{n}^{*}\right)_{n \in \mathbb{N}}\right)$ is convergent to some $y^{*} \in X^{*}$. Therefore,

$$
w^{*} \lim _{\mathcal{F}} T\left(x_{n}^{*}\right)=w^{*} \lim _{\mathcal{F}_{\mathbb{N}}} T\left(x_{n}^{*}\right)=w^{*} \lim _{n \rightarrow \infty} T\left(\left(x_{n}^{*}\right)_{n \in \mathbb{N}}\right)=\lim _{n \rightarrow \infty} T\left(\left(x_{n}^{*}\right)_{n \in \mathbb{N}}\right) .
$$

Example 2.7 The Cesàro mean operator

$T: \quad \ell_{\infty}\left(X^{*}\right) \rightarrow \ell_{\infty}\left(X^{*}\right)$,

$$
\left(x_{n}^{*}\right)_{n \in \mathbb{N}} \mapsto\left(\frac{x_{1}^{*}+\cdots+x_{n}^{*}}{n}\right)_{n \in \mathbb{N}}
$$

is an example of the previous theorem. In fact, in [7, Theorem 1] it was proved that $T_{\mathcal{F}}$ is indeed a Banach limit.

Recall that a sequence $\left(x_{n}\right)_{n \in \mathbb{N}} \subseteq \mathrm{B}_{X}$ is called a supporting sequence for an operator $T \in$ $\mathcal{B}(X, Y)$ provided that $\left\|T\left(x_{n}\right)\right\| \rightarrow\|T\|$ as $n \rightarrow \infty$. 
Example 2.8 Every continuous linear operator $T: X^{*} \rightarrow X^{*}$ induces an operator

$$
\begin{aligned}
& \widetilde{T}: \quad \ell_{\infty}\left(X^{*}\right) \mapsto \ell_{\infty}\left(X^{*}\right), \\
&\left(x_{n}^{*}\right)_{n \in \mathbb{N}} \mapsto\left(T\left(x_{n}^{*}\right)\right)_{n \in \mathbb{N}}
\end{aligned}
$$

which verifies that $\widetilde{T}\left(c\left(X^{*}\right)\right) \subseteq c\left(X^{*}\right)$ and $\lim \circ \widetilde{T}=T \circ \lim$. In fact, $\left\|\widetilde{T}_{\mathcal{F}}\right\|=\|\widetilde{T}\|=\|T\|$. Indeed, it is easy to verify that $\|\widetilde{T}\|=\|T\|$. In view of Theorem 2.6, $\left\|\widetilde{T}_{\mathcal{F}}\right\| \leq\|\widetilde{T}\|$. Let $\left(x_{n}^{*}\right)_{n \in \mathbb{N}} \subseteq \mathrm{B}_{X^{*}}$ be a supporting sequence for $T$. Observe that

$$
\widetilde{T}_{\mathcal{F}}\left(\mathbf{x}_{n}^{*}\right)=w^{*} \lim _{\mathcal{F}} \widetilde{T}\left(\mathbf{x}_{n}^{*}\right)=T\left(x_{n}^{*}\right)
$$

for all $n \in \mathbb{N}$. Thus

$$
\left\|\widetilde{T}_{\mathcal{F}}\left(\mathbf{x}_{n}^{*}\right)\right\|=\left\|T\left(x_{n}^{*}\right)\right\| \rightarrow\|T\|
$$

as $n \rightarrow \infty$. This shows that $\|T\| \leq\left\|\widetilde{T}_{\mathcal{F}}\right\|$.

Theorem 2.6 motivates the following definition.

Definition 2.9 Let $\mathcal{F}$ be a free filter of $\mathbb{N}$ or, equivalently, a filter of $\mathbb{N}$ containing the Frechet filter. Let $X$ and $Y$ be Hausdorff topological vector spaces, and let $\mathcal{V}$ and $\mathcal{W}$ be $X$ - and $Y$-sequence spaces, respectively. Consider $T \in \mathcal{C} \mathcal{L}(\mathcal{V}, \mathcal{W})$. We define the space of $(\mathcal{F}, T)$-convergence as

$$
c_{(\mathcal{F}, T)}(X):=\left\{\left(x_{n}\right)_{n \in \mathbb{N}} \in \mathcal{V}: \exists \lim _{\mathcal{F}} T\left(\left(x_{n}\right)_{n \in \mathbb{N}}\right)\right\} .
$$

The $(\mathcal{F}, T)$-limit operator is defined as

$$
\begin{aligned}
(\mathcal{F}, T) \lim : \quad c_{(\mathcal{F}, T)}(X) \rightarrow Y, \\
\left(x_{n}\right)_{n \in \mathbb{N}} \mapsto(\mathcal{F}, T) \lim x_{n}:=\lim _{\mathcal{F}} T\left(\left(x_{n}\right)_{n \in \mathbb{N}}\right) .
\end{aligned}
$$

In the previous definition, if $\mathcal{F}$ is the Frechet filter of $\mathbb{N}$, then we remove the symbol $\mathcal{F}$ and simply write $c_{T}(X)$ and $T \lim$. Also, whenever $\mathcal{V}=\mathcal{W}$ and $T$ is the identity operator, then we simply write $c_{\mathcal{F}}(X)$ and $\mathcal{F} \lim$.

Theorem 2.10 Let $\mathcal{F}$ be a free filter of $\mathbb{N}$. Let $X$ and $Y$ be Hausdorff topological vector spaces, and let $\mathcal{V}$ and $\mathcal{W}$ be $X$ - and $Y$-sequence spaces, respectively. Consider $T \in$ $\mathcal{C L}(\mathcal{V}, \mathcal{W})$. Then:

(1) $c_{(\mathcal{F}, T)}(X)$ is a subspace of $\mathcal{V}$ and $(\mathcal{F}, T) \lim$ is linear.

(2) If $\mathcal{W}$ is endowed with the uniform convergence topology, then $(\mathcal{F}, T) \lim$ is continuous.

(3) $c_{(\mathcal{F}, T)}(X)=\left\{\left(x_{n}\right)_{n \in \mathbb{N}} \in \mathcal{V}: T\left(\left(x_{n}\right)_{n \in \mathbb{N}}\right) \in c_{\mathcal{F}}(Y)\right\}=T^{-1}\left(c_{\mathcal{F}}(Y)\right)$.

(4) If $\mathcal{G}$ is another filter of $\mathbb{N}$ containing $\mathcal{F}$, then $c_{(\mathcal{F}, T)}(X) \subseteq c_{(\mathcal{G}, T)}(X)$ and $\left.(\mathcal{G}, T) \lim \right|_{c_{(\mathcal{F}, T)}(X)}=(\mathcal{F}, T) \lim$.

(5) If $c(X) \subseteq \mathcal{V}$ and $T(c(X)) \subseteq c(X)$, then $c(X) \subseteq c_{T}(X)$ and $\left.T \lim \right|_{c(X)}=\lim \circ\left(\left.T\right|_{c(X)}\right)$.

(6) If $c(X) \subseteq \mathcal{W}$ and $T^{-1}(c(X)) \subseteq c(X)$, then $c_{T}(X) \subseteq c(X)$ and $\left.\lim \right|_{c_{T}(X)}=T \mathrm{lim}$.

(7) If $X$ and $Y$ are normed, $\mathcal{V} \subseteq \ell_{\infty}(X)$ and $\mathcal{W} \subseteq \ell_{\infty}(Y)$, then $\|(\mathcal{F}, T) \lim \| \leq\|T\|$. 
(8) If $\mathcal{F}$ is a free ultrafilter, $X$ is normed, $Y$ is finite dimensional, $\mathcal{V}=\ell_{\infty}(X)$, and $\mathcal{W}=\ell_{\infty}(Y)$, then $c_{(\mathcal{F}, T)}(X)=\ell_{\infty}(X)$.

Proof

(1) Take $\left(x_{n}\right)_{n \in \mathbb{N}}$ and $\left(w_{n}\right)_{n \in \mathbb{N}}$ in $\mathcal{V}$. Denote $y:=\lim _{\mathcal{F}} T\left(\left(x_{n}\right)_{n \in \mathbb{N}}\right), z:=\lim _{\mathcal{F}} T\left(\left(w_{n}\right)_{n \in \mathbb{N}}\right)$, $\left(a_{n}\right)_{n \in \mathbb{N}}:=T\left(\left(x_{n}\right)_{n \in \mathbb{N}}\right)$, and $\left(b_{n}\right)_{n \in \mathbb{N}}:=T\left(\left(w_{n}\right)_{n \in \mathbb{N}}\right)$. Let $U$ be an open neighborhood of $y+z$ in $Y$. There are open neighborhoods $V_{y}$ and $V_{z}$ of $y$ and $z$, respectively, such that $V_{y}+V_{z} \subseteq U$. Note that $\left\{n \in \mathbb{N}: a_{n} \in V_{y}\right\} \in \mathcal{F}$ and $\left\{n \in \mathbb{N}: b_{n} \in V_{z}\right\} \in \mathcal{F}$. In particular,

$$
\left\{n \in \mathbb{N}: a_{n} \in V_{y} \text { and } b_{n} \in V_{z}\right\}=\left\{n \in \mathbb{N}: a_{n} \in V_{y}\right\} \cap\left\{n \in \mathbb{N}: b_{n} \in V_{z}\right\} \in \mathcal{F} .
$$

On the other hand,

$$
\left\{n \in \mathbb{N}: a_{n} \in V_{y} \text { and } b_{n} \in V_{z}\right\} \subseteq\left\{n \in \mathbb{N}: a_{n}+b_{n} \in U\right\},
$$

which implies that $\left\{n \in \mathbb{N}: a_{n}+b_{n} \in U\right\} \in \mathcal{F}$. Then $y+z \in \lim _{\mathcal{F}} T\left(\left(x_{n}\right)_{n \in \mathbb{N}}+\left(w_{n}\right)_{n \in \mathbb{N}}\right)$. Since $Y$ is Hausdorff, we have that $y+z=\lim _{\mathcal{F}} T\left(\left(x_{n}\right)_{n \in \mathbb{N}}+\left(w_{n}\right)_{n \in \mathbb{N}}\right)$. Now let $\lambda \in \mathbb{K}$. Let $U$ be an open neighborhood of $\lambda y$ in $Y$. There is an open neighborhood $V$ of $y$ such that $\lambda V \subseteq U$. Observe that $\left\{n \in \mathbb{N}: a_{n} \in V\right\} \in \mathcal{F}$. On the other hand,

$$
\left\{n \in \mathbb{N}: a_{n} \in V\right\} \subseteq\left\{n \in \mathbb{N}: \lambda a_{n} \in \lambda V\right\} \subseteq\left\{n \in \mathbb{N}: \lambda a_{n} \in U\right\}
$$

which implies $\left\{n \in \mathbb{N}: \lambda a_{n} \in U\right\} \in \mathcal{F}$. This shows that $\lambda y \in \lim _{\mathcal{F}}\left(\lambda a_{n}\right)=\lim _{\mathcal{F}} T\left(\left(\lambda x_{n}\right)_{n \in \mathbb{N}}\right)$. Since $Y$ is Hausdorff, we have that $\lambda y=\lim _{\mathcal{F}} T\left(\left(\lambda x_{n}\right)_{n \in \mathbb{N}}\right)$.

(2) Let $U$ be a neighborhood of 0 in $Y$. Since all topological vector spaces are regular, we may assume without loss that $U$ is closed. Take $W:=U^{\mathbb{N}} \cap \mathcal{W}$, which is a neighborhood of 0 in $\mathcal{W}$. Take $V:=T^{-1}(W)$, which is a neighborhood of 0 in $\mathcal{V}$. We will show that $(\mathcal{F}, T) \lim (V) \subseteq U$. Let $\left(x_{n}\right)_{n \in \mathbb{N}} \in V$. Then $\left(y_{n}\right)_{n \in \mathbb{N}}:=T\left(\left(x_{n}\right)_{n \in \mathbb{N}}\right) \in W$. Denote $y:=\lim _{\mathcal{F}} y_{n}=(\mathcal{F}, T) \lim x_{n}$. Suppose that $y \notin U$. Since $U$ is closed, we can find an open neighborhood $A$ of $y$ such that $A \cap U=\varnothing$. Note that $\left\{n \in \mathbb{N}: y_{n} \in A\right\} \in \mathcal{F}$. Since $\left(y_{n}\right)_{n \in \mathbb{N}} \in W$, we have that $y_{n} \in U$ for every $n \in \mathbb{N}$. Since $U \cap A=\varnothing$, we conclude that $\left\{n \in \mathbb{N}: y_{n} \in A\right\}=\varnothing$, which implies the contradiction that $\varnothing \in \mathcal{F}$.

(3) Trivial.

(4) Simply observe that since $\mathcal{F} \subseteq \mathcal{G}$, we have that $\lim _{\mathcal{F}} T\left(\left(x_{n}\right)_{n \in \mathbb{N}}\right) \subseteq \lim _{\mathcal{G}} T\left(\left(x_{n}\right)_{n \in \mathbb{N}}\right)$, which implies that $c_{(\mathcal{F}, T)}(X) \subseteq c_{(\mathcal{G}, T)}(X)$. The Hausdorff character of $X$ implies that $\left.(\mathcal{G}, T) \lim \right|_{c_{(\mathcal{F}, T)}(X)}=(\mathcal{F}, T) \lim$.

(5) If $\left(x_{n}\right)_{n \in \mathbb{N}} \in c(X)$, then by hypothesis we have that $T\left(\left(x_{n}\right)_{n \in \mathbb{N}}\right) \in c(X)$, so

$$
\lim _{n \rightarrow \infty} T\left(\left(x_{n}\right)_{n \in \mathbb{N}}\right)=\lim _{\mathcal{F}_{\mathbb{N}}} T\left(\left(x_{n}\right)_{n \in \mathbb{N}}\right)=T \lim x_{n},
$$

which implies that $\left(x_{n}\right)_{n \in \mathbb{N}} \in c_{T}(X)$ and $\left.T \lim \right|_{c(X)}=\lim \circ\left(\left.T\right|_{c(X)}\right)$. 
(6) If $\left(x_{n}\right)_{n \in \mathbb{N}} \in c_{T}(X)$, then $\lim _{\mathcal{F}_{\mathbb{N}}} T\left(\left(x_{n}\right)_{n \in \mathbb{N}}\right)$ exists, but we know that $\lim _{\mathcal{F}_{\mathbb{N}}} T\left(\left(x_{n}\right)_{n \in \mathbb{N}}\right)=\lim _{n \rightarrow \infty} T\left(\left(x_{n}\right)_{n \in \mathbb{N}}\right)$. As a consequence, $T\left(\left(x_{n}\right)_{n \in \mathbb{N}}\right) \in c(X)$. Then, by hypothesis, $\left(x_{n}\right)_{n \in \mathbb{N}} \in c(X)$ and $\left.\lim \right|_{c_{T}(X)}=T \lim$.

(7) If $\left(x_{n}\right)_{n \in \mathbb{N}} \in \mathrm{B}_{\ell_{\infty}(X)} \cap \mathcal{V}=\mathrm{B}_{\mathcal{V}}$, then

$$
T\left(\left(x_{n}\right)_{n \in \mathbb{N}}\right) \in \mathrm{B}_{\ell_{\infty}(Y)}\left(0,\left\|T\left(\left(x_{n}\right)_{n \in \mathbb{N}}\right)\right\|_{\infty}\right) \subseteq \mathrm{B}_{\ell_{\infty}(Y)}(0,\|T\|),
$$

which implies that $\lim _{\mathcal{F}} T\left(\left(x_{n}\right)_{n \in \mathbb{N}}\right) \in \mathrm{B}_{Y}(0,\|T\|)$, and hence $\|(\mathcal{F}, T) \lim \| \leq\|T\|$.

(8) In the first place, note that if $Y$ is a finite dimensional Hausdorff topological vector space, then $Y$ is normed. Let $\left(x_{n}\right)_{n \in \mathbb{N}} \in \ell_{\infty}(X)$. Note that $\mathrm{B}_{Y}\left(0,\left\|T\left(\left(x_{n}\right)_{n \in \mathbb{N}}\right)\right\|_{\infty}\right)$ is compact. Denote $\left(a_{n}\right)_{n \in \mathbb{N}}:=T\left(\left(x_{n}\right)_{n \in \mathbb{N}}\right)$. Then $\mathcal{G}:=\left\{\left\{a_{n}: n \in F\right\}: F \in \mathcal{F}\right\}$ is a filter base in $\mathrm{B}_{Y}\left(0,\left\|T\left(\left(x_{n}\right)_{n \in \mathbb{N}}\right)\right\|_{\infty}\right)$ whose induced filter is an ultrafilter in view of Remark 2.2. The compactness of $\mathrm{B}_{Y}\left(0,\left\|T\left(\left(x_{n}\right)_{n \in \mathbb{N}}\right)\right\|_{\infty}\right)$ allows that $\lim (\mathcal{G})=\lim (\mathcal{J}(\mathcal{G})) \neq \varnothing$. Finally, notice that $\lim _{(\mathcal{F}, T)} x_{n}=\lim _{\mathcal{F}} T\left(\left(x_{n}\right)_{n \in \mathbb{N}}\right)=\lim (\mathcal{G})$.

Theorem 2.10(8) can be in fact proved easily by relying on Theorem 2.3(5). Indeed, by Theorem 2.10(3),

$$
c_{(\mathcal{F}, T)}(X)=\left\{\left(x_{n}\right)_{n \in \mathbb{N}} \in \ell_{\infty}(X): T\left(\left(x_{n}\right)_{n \in \mathbb{N}}\right) \in c_{\mathcal{F}}(Y)\right\}=T^{-1}\left(c_{\mathcal{F}}(Y)\right) .
$$

In the settings of Theorem 2.10(8), we have that $c_{\mathcal{F}}(Y)=\ell_{\infty}(Y)$ by virtue of Theorem 2.3(5). As a consequence,

$$
c_{(\mathcal{F}, T)}(X)=T^{-1}\left(c_{\mathcal{F}}(Y)\right)=T^{-1}\left(\ell_{\infty}(Y)\right)=\ell_{\infty}(X) .
$$

Definition 2.9 allows us to describe the vector space of Cauchy sequences.

Example 2.11 Let $X$ be a normed space, and let us denote by $\bar{X}$ its completion. It is clear that the vector space of Cauchy sequences on $X$ is described by $c(\bar{X}) \cap \ell_{\infty}(X)$. If we let $\iota_{X}: \ell_{\infty}(X) \rightarrow \ell_{\infty}(\bar{X})$ denote the canonical inclusion, then $c_{l_{X}}(X)=c(\bar{X}) \cap \ell_{\infty}(X)$.

We will describe now the almost convergence in terms of the $(\mathcal{F}, T)$-convergence.

Example 2.12 Let $X$ be a Hausdorff topological vector space. The general $k$-Cesàro mean operator is defined as

$$
\begin{aligned}
\mathscr{C}_{k}: & X^{\mathbb{N}} \rightarrow X^{\mathbb{N}}, \\
& \left(x_{n}\right)_{n \in \mathbb{N}} \mapsto \mathscr{C}_{k}\left(\left(x_{n}\right)_{n \in \mathbb{N}}\right):=\left(\frac{x_{k}+\cdots+x_{k+n-1}}{n}\right)_{n \in \mathbb{N}} .
\end{aligned}
$$

Observe that if $X$ is locally convex, then $\mathscr{C}_{k}\left(\ell_{\infty}(X)\right) \subseteq \ell_{\infty}(X)$. The general uniform Cesàro mean operator is defined as

$$
\begin{array}{ll}
\mathscr{C}: & X^{\mathbb{N}} \rightarrow\left(X^{\mathbb{N}}\right)^{\mathbb{N}} \\
& \left(x_{n}\right)_{n \in \mathbb{N}} \mapsto \mathscr{C}\left(\left(x_{n}\right)_{n \in \mathbb{N}}\right):=\left(C_{k}\left(\left(x_{n}\right)_{n \in \mathbb{N}}\right)\right)_{k \in \mathbb{N}} .
\end{array}
$$


Observe that if $X$ is locally convex and $\ell_{\infty}(X)$ is endowed with the uniform convergence topology, then $\mathscr{C}\left(\ell_{\infty}(X)\right) \subseteq \ell_{\infty}\left(\ell_{\infty}(X)\right)$.

Connected with the previous example, note that applications of almost convergence to nonlinear ergodic theory can be found in $[8,19]$.

Lemma 2.13 Let $X$ be a Hausdorff locally convex topological vector space. If $\ell_{\infty}(X)$ is endowed with the uniform convergence topology, then $\mathscr{C}\left(\ell_{\infty}(X)\right) \subseteq \ell_{\infty}\left(\ell_{\infty}(X)\right)$.

Proof Fix arbitrary $\left(x_{n}\right)_{n \in \mathbb{N}} \in \ell_{\infty}(X)$. We will show first that $\left(\frac{x_{k}+\cdots+x_{k+n-1}}{n}\right)_{n \in \mathbb{N}} \in \ell_{\infty}(X)$. Let $V$ be a convex zero neighborhood in $X$. There exists $\alpha \in \mathbb{K}$ such that $\left(x_{n}\right)_{n \in \mathbb{N}} \subseteq \alpha V$. Since $\alpha V$ is convex, we have that $\left(\frac{x_{k}+\cdots+x_{k+n-1}}{n}\right)_{n \in \mathbb{N}} \subseteq \alpha V$ for all $k \in \mathbb{N}$. This shows that $\left(\frac{x_{k}+\cdots+x_{k+n-1}}{n}\right)_{n \in \mathbb{N}} \in \ell_{\infty}(X)$. Finally, let us show that $\left(\left(\frac{x_{k}+\cdots+x_{k+n-1}}{n}\right)_{n \in \mathbb{N}}\right)_{k \in \mathbb{N}} \in \ell_{\infty}\left(\ell_{\infty}(X)\right)$. Take $U$ a zero neighborhood in $\ell_{\infty}(X)$. Since $\ell_{\infty}(X)$ is endowed with the uniform convergence topology, we can find a convex zero neighborhood $V$ in $X$ such that $V^{\mathbb{N}} \cap \ell_{\infty}(X) \subseteq$ $U$. Again, take $\alpha \in \mathbb{K}$ such that $\left(x_{n}\right)_{n \in \mathbb{N}} \subseteq \alpha V$. We know that $\left(\frac{x_{k}+\cdots+x_{k+n-1}}{n}\right)_{n \in \mathbb{N}} \subseteq \alpha V$ for all $k \in \mathbb{N}$, that is, $\left(\frac{x_{k}+\cdots+x_{k+n-1}}{n}\right)_{n \in \mathbb{N}} \in \alpha V^{\mathbb{N}} \cap \ell_{\infty}(X) \subseteq \alpha U$ for all $k \in \mathbb{N}$. Therefore, $\left(\left(\frac{x_{k}+\cdots+x_{k+n-1}}{n}\right)_{n \in \mathbb{N}}\right)_{k \in \mathbb{N}} \subseteq \alpha U$. This shows that $\left(\left(\frac{x_{k}+\cdots+x_{k+n-1}}{n}\right)_{n \in \mathbb{N}}\right)_{k \in \mathbb{N}} \in \ell_{\infty}\left(\ell_{\infty}(X)\right)$.

Lemma 2.14 Let $X$ be a Hausdorff topological vector space. Let $\left(x_{n}\right)_{n \in \mathbb{N}}$ be a sequence in $X$. If there exists $k \in \mathbb{N}$ such that $\mathscr{C}_{k}\left(\left(x_{n}\right)_{n \in \mathbb{N}}\right)$ is convergent, then $\mathscr{C}_{l}\left(\left(x_{n}\right)_{n \in \mathbb{N}}\right)$ is convergent to the same limit for all $l \in \mathbb{N}$. In particular, if $\mathscr{C}\left(\left(x_{n}\right)_{n \in \mathbb{N}}\right)$ is pointwise convergent, then the limit is a constant sequence.

Proof We will assume that $k>1$ and show that $\mathscr{C}_{1}\left(\left(x_{n}\right)_{n \in \mathbb{N}}\right)$ is convergent to the same limit. Observe that

$$
\frac{x_{1}+\cdots+x_{k+n-1}}{k+n-1}=\frac{k-1}{k+n-1} \frac{x_{1}+\cdots+x_{k-1}}{k-1}+\frac{n}{k+n-1} \frac{x_{k}+\cdots+x_{k+n-1}}{n}
$$

for all $n \in \mathbb{N}$. If we let $n \rightarrow \infty$, then

$$
\frac{k-1}{k+n-1} \frac{x_{1}+\cdots+x_{k-1}}{k-1} \rightarrow 0
$$

since $k$ is fixed, which shows that

$$
\lim _{n \rightarrow \infty} \frac{x_{1}+\cdots+x_{k+n-1}}{k+n-1}=\lim _{n \rightarrow \infty} \frac{x_{k}+\cdots+x_{k+n-1}}{n} .
$$

Now that we know that $\mathscr{C}_{1}\left(\left(x_{n}\right)_{n \in \mathbb{N}}\right)$ is convergent to the same limit, we will show that $\mathscr{C}_{l}\left(\left(x_{n}\right)_{n \in \mathbb{N}}\right)$ is convergent to the same limit for all $l \in \mathbb{N}$. Fix arbitrary $l>1$. By relying on the same expression as in (2.1), we have that

$$
\frac{x_{1}+\cdots+x_{l+n-1}}{l+n-1}=\frac{l-1}{l+n-1} \frac{x_{1}+\cdots+x_{l-1}}{l-1}+\frac{n}{l+n-1} \frac{x_{l}+\cdots+x_{l+n-1}}{n}
$$

for all $n \in \mathbb{N}$. By isolating $\frac{n}{l+n-1} \frac{x_{l}+\cdots+x_{l+n-1}}{n}$ from (2.2) we conclude that $\mathscr{C}_{l}\left(\left(x_{n}\right)_{n \in \mathbb{N}}\right)$ is convergent to the same limit as $\mathscr{C}_{1}\left(\left(x_{n}\right)_{n \in \mathbb{N}}\right)$. 
Theorem 2.15 If $X$ is a Hausdorff locally convex topological vector space and $\ell_{\infty}(X)$ is endowed with the uniform convergence topology, then $\operatorname{ac}(X)=c_{\mathscr{C}_{\ell_{\infty}(X)}}(X)$, where $\mathscr{C}_{\ell_{\infty}(X)}$ is the general Cesàro operator restricted to $\ell_{\infty}(X)$ with range in $\ell_{\infty}\left(\ell_{\infty}(X)\right)$.

Proof In the first place, since $X$ is locally convex, we have that $\mathscr{C}_{k}\left(\ell_{\infty}(X)\right) \subseteq \ell_{\infty}(X)$ for all $k \in \mathbb{N}$ as we remarked in Example 2.12. Also keep in mind that, in view of Lemma 2.14, if $\mathscr{C}\left(\left(x_{n}\right)_{n \in \mathbb{N}}\right)$ is pointwise convergent, then the limit is a constant sequence. Notice also that $\ell_{\infty}(X)$ is endowed with the uniform convergence topology. With all these ingredients we conclude that

$$
\begin{aligned}
c_{\left.\mathscr{C}\right|_{\ell_{\infty}(X)}}(X): & :\left\{\left(x_{n}\right)_{n \in \mathbb{N}} \in \ell_{\infty}(X): \exists \lim _{\mathcal{F}_{\mathbb{N}}} \mathscr{C}\left(\left(x_{n}\right)_{n \in \mathbb{N}}\right)\right\} \\
: & \left\{\left(x_{n}\right)_{n \in \mathbb{N}} \in \ell_{\infty}(X): \mathscr{C}\left(\left(x_{n}\right)_{n \in \mathbb{N}}\right) \in c\left(\ell_{\infty}(X)\right)\right\} \\
:= & \left\{\left(x_{n}\right)_{n \in \mathbb{N}} \in \ell_{\infty}(X): \exists x \in X \text { such that } \lim _{\mathcal{F}_{\mathbb{N}}} \mathscr{C}\left(\left(x_{n}\right)_{n \in \mathbb{N}}\right)=\mathbf{x}\right\} \\
= & \left\{\left(x_{n}\right)_{n \in \mathbb{N}} \in \ell_{\infty}(X): \exists x \in X\right. \text { such that } \\
& \left.\lim _{n \rightarrow \infty} \frac{x_{k}+\cdots+x_{k+n-1}}{n}=x \text { uniformly in } k \in \mathbb{N}\right\} \\
= & \operatorname{ac}(X) .
\end{aligned}
$$

\subsection{Multipliers}

The concept of multiplier convergent series has been widely studied since the beginning of this century and basically allows to describe the behavior of a series through a certain space of sequences. We refer the reader to the magnificent book [21] on multiplier convergent series. In [22] the vector-valued version of multipliers was introduced for the first time. For recent developments on this topic, see $[6,15,16]$. Here we adapt it to our general method of convergence.

Definition 2.16 Let $\mathcal{G}$ be a free filter of $\mathbb{N}$. Let $X, Y$, and $Z$ be Hausdorff topological vector spaces. Let $\mathcal{U}, \mathcal{V}$, and $\mathcal{W}$ be $X$-, $Y$-, and $Z$-sequence spaces, respectively. Consider $S \in$ $\mathcal{C} \mathcal{L}(\mathcal{V}, \mathcal{W})$. Then:

(1) The $(\mathcal{G}, S)$-multiplier space associated with a sequence $\left(T_{n}\right)_{n \in \mathbb{N}} \subseteq \mathcal{C} \mathcal{L}(X, Y)$ is defined as

$$
\mathcal{M}_{(\mathcal{G}, S)}^{\infty}\left(\left(T_{n}\right)_{n \in \mathbb{N}}\right):=\left\{\left(x_{n}\right)_{n \in \mathbb{N}} \in \mathcal{U}: \sum_{n=1}^{\infty} T_{n}\left(x_{n}\right) \in c_{(\mathcal{G}, S)}(Y)\right\}
$$

and the $(\mathcal{G}, S)$-summing operator associated with $\left(T_{n}\right)_{n \in \mathbb{N}}$ is defined as

$$
\begin{aligned}
(\mathcal{G}, S) \sum_{n=1}^{\infty} T_{n}: & \mathcal{M}_{(\mathcal{G}, S)}^{\infty}\left(\left(T_{n}\right)_{n \in \mathbb{N}}\right) \rightarrow Z, \\
& \left(x_{n}\right)_{n \in \mathbb{N}} \mapsto(\mathcal{G}, S) \sum_{n=1}^{\infty} T_{n}\left(x_{n}\right) .
\end{aligned}
$$


(2) The $(\mathcal{G}, S)$-summability space associated with a subspace $\mathcal{S} \subseteq \mathcal{V}$ is defined as

$$
\mathcal{C} \mathcal{L}(X, Y)(\mathcal{S}):=\left\{\left(T_{n}\right)_{n \in \mathbb{N}} \in \mathcal{C} \mathcal{L}(X, Y)^{\mathbb{N}}: \mathcal{S} \subseteq \mathcal{M}_{(\mathcal{G}, S)}^{\infty}\left(\left(T_{n}\right)_{n \in \mathbb{N}}\right)\right\}
$$

In the previous definition, if $\mathcal{G}$ is the Frechet filter of $\mathbb{N}$, then we remove the symbol $\mathcal{G}$ and simply write $\mathcal{M}_{S}^{\infty}\left(\left(T_{n}\right)_{n \in \mathbb{N}}\right)$ and $S \sum_{n=1}^{\infty} T_{n}$. Also, whenever $\mathcal{V}=\mathcal{W}$ and $S$ is the identity operator, then we simply write $\mathcal{M}_{\mathcal{G}}^{\infty}\left(\left(T_{n}\right)_{n \in \mathbb{N}}\right)$ and $\mathcal{G} \sum_{n=1}^{\infty} T_{n}$.

Lemma 2.17 Let $Z$ be a normed space. If $\left(z_{n}\right)_{n \in \mathbb{N}}$ is a Cauchy sequence in $Z$ which is weakly convergent to some $z \in Z$, then $\left(z_{n}\right)_{n \in \mathbb{N}}$ is norm-convergent to $z$. In other words, $c_{l Z}(Z) \cap$ $c\left(Z_{w}\right)=c(Z)$, where $\iota_{Z}: \ell_{\infty}(Z) \rightarrow \ell_{\infty}(\bar{Z})$ denotes the canonical inclusion and $Z_{w}$ stands for $Z$ endowed with the weak topology.

Proof Notice that $\left(z_{n}\right)_{n \in \mathbb{N}}$ is also weakly convergent to $z$ in $\bar{Z}$. On the other hand, there exists $z_{0} \in \bar{Z}$ such that $\left(z_{n}\right)_{n \in \mathbb{N}}$ converges to $z_{0}$ in $\bar{Z}$. Then $z_{0}=z$ and so $z_{0} \in Z$. This proves the result.

In the next result of this subsection, $\mathcal{G}$ will be the Frechet filter of $\mathbb{N}, \mathcal{F}_{\mathbb{N}}, X, Y$, and $Z$ will be normed spaces and $\mathcal{U}:=\ell_{\infty}(X), \mathcal{V}:=\ell_{\infty}(Y)$, and $\mathcal{W}:=\ell_{\infty}(Z)$, endowed with the sup norm.

Proposition 2.18 Let $X, Y$, and $Z$ be normed spaces and $S \in \mathcal{B}\left(\ell_{\infty}(Y), \ell_{\infty}(Z)\right)$ such that $S\left(c_{l Y}(Y)\right) \subseteq c_{l Z}(Z)$. Let $Z_{w}$ denote $Z$ endowed with the weak topology, and let $S_{w}$ denote $S$ seen as a continuous linear operator from $\ell_{\infty}(Y)$ to $\ell_{\infty}\left(Z_{w}\right)$. Consider a sequence $\left(T_{n}\right)_{n \in \mathbb{N}} \subseteq$ $\mathcal{B}(X, Y)$. If $\ell_{\infty}(X) \subseteq \mathcal{M}_{l_{Y}}^{\infty}\left(\left(T_{n}\right)_{n \in \mathbb{N}}\right)$, then $\mathcal{M}_{S}^{\infty}\left(\left(T_{n}\right)_{n \in \mathbb{N}}\right)=\mathcal{M}_{S_{w}}^{\infty}\left(\left(T_{n}\right)_{n \in \mathbb{N}}\right)$.

Proof Since the weak topology is coarser than the norm topology on $Z$, we have that $c_{S}(Y) \subseteq c_{S_{w}}(Y)$. Hence $\mathcal{M}_{S}^{\infty}\left(\left(T_{n}\right)_{n \in \mathbb{N}}\right) \subseteq \mathcal{M}_{S_{w}}^{\infty}\left(\left(T_{n}\right)_{n \in \mathbb{N}}\right)$. Conversely, take $\left(x_{n}\right)_{n \in \mathbb{N}} \in$ $\mathcal{M}_{S_{w}}^{\infty}\left(\left(T_{n}\right)_{n \in \mathbb{N}}\right)$. By hypothesis, $\sum_{n=1}^{\infty} T_{n}\left(x_{n}\right) \in c_{l Y}(Y)$ so $S\left(\sum_{n=1}^{\infty} T_{n}\left(x_{n}\right)\right) \in c_{l Z}(Z)$. However, $S\left(\sum_{n=1}^{\infty} T_{n}\left(x_{n}\right)\right) \in c\left(Z_{w}\right)$. Finally, by Lemma 2.17, we conclude that $S\left(\sum_{n=1}^{\infty} T_{n}\left(x_{n}\right)\right) \in c(Z)$. This shows that $\left(x_{n}\right)_{n \in \mathbb{N}} \in \mathcal{M}_{S}^{\infty}\left(\left(T_{n}\right)_{n \in \mathbb{N}}\right)$.

The following final lemma shows that the general Cesàro operator verifies the hypothesis of the previous proposition.

Lemma 2.19 Let $X$ be a normed space. Consider $\left.\mathscr{C}\right|_{\ell_{\infty}(X)}$ the general Cesàro operator restricted to $\ell_{\infty}(X)$ with range in $\ell_{\infty}\left(\ell_{\infty}(X)\right)$. Let $\left(x_{n}\right)_{n \in \mathbb{N}}$ be a bounded sequence in $X$. If there exists $k \in \mathbb{N}$ such that $\mathscr{C}_{k}\left(\left(x_{n}\right)_{n \in \mathbb{N}}\right)$ is Cauchy, then $\mathscr{C}_{l}\left(\left(x_{n}\right)_{n \in \mathbb{N}}\right)$ is Cauchy for all $l \in \mathbb{N}$. In particular, if $\left(x_{n}\right)_{n \in \mathbb{N}}$ is a Cauchy sequence in $X$, then $\mathscr{C}\left(\left(x_{n}\right)_{n \in \mathbb{N}}\right)$ is Cauchy in $\ell_{\infty}(X)$.

Proof Note that $\mathscr{C}_{k}\left(\left(x_{n}\right)_{n \in \mathbb{N}}\right)$ is convergent in $\bar{X}$. So if we apply Lemma 2.14 in $\bar{X}$, then we conclude that $\mathscr{C}_{l}\left(\left(x_{n}\right)_{n \in \mathbb{N}}\right)$ is convergent and thus Cauchy for all $l \in \mathbb{N}$. Now if $\left(x_{n}\right)_{n \in \mathbb{N}}$ is a Cauchy sequence in $X$, then $\left(x_{n}\right)_{n \in \mathbb{N}}$ is convergent in $\bar{X}$, so it is almost convergent in $\bar{X}$, that is, $\mathscr{C}\left(\left(x_{n}\right)_{n \in \mathbb{N}}\right) \in c\left(\ell_{\infty}(\bar{X})\right)$, which implies that $\mathscr{C}\left(\left(x_{n}\right)_{n \in \mathbb{N}}\right)$ is Cauchy in $\ell_{\infty}(X)$.

\section{Convergence through operators}

This section is strongly motivated by the vector-valued Banach limit theory, which can be found in $[1,2,7,13,14]$. 


\subsection{The set $\mathcal{H} \mathcal{B}(\lim )$}

Recall that if $X$ and $Y$ are normed spaces and $S \in \mathcal{B}(W, Y)$ where $W$ is a subspace of $X$, then we can define the set

$$
\mathcal{H B}(S):=\left\{T \in \mathcal{B}(X, Y):\left.T\right|_{W}=S \text { and }\|T\|=\|S\|\right\}
$$

Following this notation we have that

$$
\mathcal{H B}(\lim ):=\left\{T \in \mathcal{B}\left(\ell_{\infty}(X), X\right):\left.T\right|_{c(X)}=\lim \text { and }\|T\|=1\right\} .
$$

If there is confusion with the space $X$, then we will use the notation $\mathcal{H} \mathcal{B}_{X}(\lim )$. Notice that $\mathcal{H B}(\lim )$ is a convex subset of $S_{\mathcal{B}\left(\ell_{\infty}(X), X\right)}$ which is closed for the pointwise convergence topology of $\mathcal{B}\left(\ell_{\infty}(X), X\right)$, that is, $\sigma\left(\mathcal{B}\left(\ell_{\infty}(X), X\right),\left\{\delta_{\left(x_{n}\right)_{n \in \mathbb{N}}}:\left(x_{n}\right)_{n \in \mathbb{N}} \in \ell_{\infty}(X)\right\}\right)$.

Let us study the extremal structure of $\mathcal{H} \mathcal{B}(\lim )$. For this, we have to introduce a bit of notation. We denote

$$
\mathcal{L}_{X}^{0}:=\left\{T \in \mathcal{B}\left(\ell_{\infty}(X), X\right): c_{0}(X) \subseteq \operatorname{ker}(T)\right\}
$$

Notice that $\mathcal{L}_{X}^{0}$ is a vector subspace of $\mathcal{B}\left(\ell_{\infty}(X), X\right)$ which is closed for the pointwise convergence topology.

Recall that, for every $x \in X, \mathbf{x}$ stands for the constant sequence of general term $x$ and $\mathbf{X}$ means the space of all $\mathbf{x s .}$

A subset $E$ of a subset $C$ of a real vector space $X$ is said to be extremal if $E$ verifies the extremal condition with respect to $C$ : if $x, y \in C$ and $t \in(0,1)$ and $t x+(1-t) y \in E$, then $x, y \in E$. If $C$ is convex and $E$ is convex and extremal in $C$, then $E$ is called a face of $C$. We refer the reader to $[3,9,10]$.

Theorem 3.1 Let $X$ be a normed space with the Bade property, that is, $\mathrm{B}_{X}=\overline{\mathrm{co}}\left(\operatorname{ext}\left(\mathrm{B}_{X}\right)\right)$. Then $\mathcal{H} \mathcal{B}(\mathrm{lim})$ is a face of $\mathrm{B}_{\mathcal{L}_{X}^{0}}$.

Proof Let $T, S \in \mathrm{B}_{\mathcal{L}_{X}^{0}}$ and $t \in(0,1)$ such that $t T+(1-t) S \in \mathcal{H} \mathcal{B}(\lim )$. If $x \in \operatorname{ext}\left(\mathrm{B}_{X}\right)$, then $x=(t T+(1-t) S)(\mathbf{x})=t T(\mathbf{x})+(1-t) S(\mathbf{x})$, which implies that $T(\mathbf{x})=S(\mathbf{x})=x$. Since $\mathrm{B}_{X}=$ $\overline{\mathrm{co}}\left(\operatorname{ext}\left(\mathrm{B}_{X}\right)\right)$, we conclude that $T(\mathbf{x})=S(\mathbf{x})=x$ for all $x \in X$. Since $T, S \in \mathcal{L}_{X}^{0}$, we conclude that $T, S \in \mathcal{H B}(\mathrm{lim})$.

In [14, Theorem 5.1] it was proved that if $X$ has the Bade property, then the set of Banach limits, $\mathscr{B} \mathscr{L}(X):=\mathcal{H} \mathcal{B}(\lim ) \cap \mathcal{N}_{X}$ (see [7, Definition 2]), is a face of $\mathrm{B}_{\mathcal{N}_{X}}$, where

$$
\mathcal{N}_{X}:=\left\{T \in \mathcal{B}\left(\ell_{\infty}(X), X\right): T\left(\left(x_{n}\right)_{n \in \mathbb{N}}\right)=T\left(\left(x_{n+1}\right)_{n \in \mathbb{N}}\right) \text { for all }\left(x_{n}\right)_{n \in \mathbb{N}} \in \ell_{\infty}(X)\right\}
$$

Notice that $\mathcal{L}_{X}^{0}$ is a vector subspace of $\mathcal{N}_{X}$, and $\mathcal{N}_{X}$ is a vector subspace of $\mathcal{B}\left(\ell_{\infty}(X), X\right)$ which is closed for the pointwise convergence topology. In [1, Lemma 2.2] it was proved that the vector space of bounded sequences with bounded partial sums,

$$
\operatorname{bps}(X):=\left\{\left(x_{n}\right)_{n \in \mathbb{N}} \in X^{\mathbb{N}}:\left(\sum_{n=1}^{k} x_{n}\right)_{k \in \mathbb{N}} \in \ell_{\infty}(X)\right\},
$$


can be expressed as $\operatorname{bps}(X)=\left\{\left(z_{n+1}-z_{n}\right)_{n \in \mathbb{N}}:\left(z_{n}\right)_{n \in \mathbb{N}} \in \ell_{\infty}(X)\right\}$. As a consequence, $\mathcal{N}_{X}=$ $\left\{T \in \mathcal{B}\left(\ell_{\infty}(X), X\right): \operatorname{bps}(X) \subseteq \operatorname{ker}(T)\right\}$. Since $c_{00}(X) \subseteq \operatorname{bps}(X), c_{00}(X)$ is dense in $c(X)$ and $\operatorname{ker}(T)$ is closed, we conclude that $\mathcal{N}_{X} \subseteq \mathcal{L}_{X}^{0}$.

Now, by bearing in mind the fact that if $C$ is a face of $\mathrm{B}_{X}$ and $Y$ is a subspace of $X$, then $C \cap Y$ is a face of $\mathrm{B}_{Y}$, we have that [14, Theorem 5.1] is a direct consequence of our Theorem 3.1.

Corollary 3.2 ([14]) Let $X$ be a normed space with the Bade property. Then $\mathscr{B} \mathscr{L}(X)$ is a face of $\mathrm{B}_{\mathcal{N}_{X}}$.

Proof By Theorem 3.1 we know that $\mathcal{H B}(\lim )$ is a face of $\mathrm{B}_{\mathcal{L}_{X}^{0}}$. Thus $\mathscr{B} \mathscr{L}(X)=\mathcal{H} \mathcal{B}(\lim ) \cap$ $\mathcal{N}_{X}$ is a face of $\mathrm{B}_{\mathcal{N}_{X}}$.

We will prove next that, for certain Banach spaces, $\mathcal{N}_{X} \subsetneq \mathcal{L}_{X}^{0}$.

Theorem 3.3 Let $X$ be an injective Banach space. Then $\mathcal{N}_{X} \subsetneq \mathcal{L}_{X}^{0}$.

Proof Fix $x \in X \backslash\{0\}$ and consider the map

$$
\begin{aligned}
& c_{0}(X) \oplus \mathbb{K}\left((-1)^{n} x\right)_{n \in \mathbb{N}} \rightarrow X, \\
& \left(x_{n}\right)_{n \in \mathbb{N}}+\lambda\left((-1)^{n} x\right)_{n \in \mathbb{N}} \mapsto \lambda x .
\end{aligned}
$$

Notice that

$$
\|\lambda x\| \leq \sup _{k \in \mathbb{N}}\left\|x_{2 k}+\lambda x\right\| \leq \sup _{n \in \mathbb{N}}\left\|x_{n}+\lambda(-1)^{n} x\right\|=\left\|\left(x_{n}\right)_{n \in \mathbb{N}}+\lambda\left((-1)^{n} x\right)_{n \in \mathbb{N}}\right\|_{\infty} .
$$

This shows that the above operator has norm 1 . By hypothesis, it can be extended to the whole of $\ell_{\infty}(X)$. This extension is clearly an element of $\mathcal{L}_{X}^{0} \backslash \mathcal{N}_{X}$ since $\left((-1)^{n} x\right)_{n \in \mathbb{N}} \in$ $\operatorname{bps}(X)$.

A supporting sequence $\left(x_{n}\right)_{n \in \mathbb{N}}$ for an operator $T$ of norm 1 is called self-supporting if $x_{n}-T\left(x_{n}\right) \rightarrow 0$ as $n \rightarrow \infty$.

A convex component is a maximal convex subset (see [12]).

Theorem 3.4 Let $X$ be a normed space. If there exists $T \in \mathcal{G}_{X} \backslash\left\{I_{X}\right\}$ with a self-supporting sequence $\left(x_{n}\right)_{n \in \mathbb{N}} \subseteq \mathrm{B}_{X}$, then $\mathcal{H} \mathcal{B}(\mathrm{lim})$ is not a convex component of $\mathrm{S}_{\mathcal{L}_{0}^{X}}$.

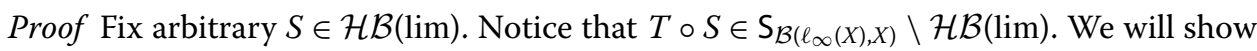
that $\mathcal{H B}(\lim ) \subsetneq \operatorname{co}(\mathcal{H B}(\lim ) \cup\{T \circ S\}) \subseteq \mathrm{S}_{\mathcal{B}\left(\ell_{\infty}(X), X\right)}$. We already know that $\mathcal{H B}(\lim ) \subsetneq$ $\operatorname{co}(\mathcal{H B}(\lim ) \cup\{T \circ S\})$. Let $R \in \mathcal{H B}(\lim )$ and $t \in(0,1)$. It is clear that $t R+(1-t)(T \circ S) \in \mathrm{B}_{\mathcal{L}_{X}^{0}}$. All is left to prove is that $\|t R+(1-t)(T \circ S)\|=1$. Simply notice that $(t R+(1-t)(T \circ S))\left(\mathbf{x}_{n}\right)=$ $t x_{n}+(1-t) T\left(x_{n}\right)=t\left(x_{n}-T\left(x_{n}\right)\right)+T\left(x_{n}\right)$ for all $n \in \mathbb{N}$, which implies that

$$
\begin{aligned}
\left|\left\|(t R+(1-t)(T \circ S))\left(\mathbf{x}_{n}\right)\right\|-\left\|T\left(x_{n}\right)\right\|\right| & \leq\left\|(t R+(1-t)(T \circ S))\left(\mathbf{x}_{n}\right)-T\left(x_{n}\right)\right\| \\
& =\left\|t\left(x_{n}-T\left(x_{n}\right)\right)+T\left(x_{n}\right)-T\left(x_{n}\right)\right\| \\
& =t\left\|x_{n}-T\left(x_{n}\right)\right\| \rightarrow 0 .
\end{aligned}
$$


Since $\left(\left\|T\left(x_{n}\right)\right\|\right)_{n \in \mathbb{N}}$ converges to 1 , we conclude that $\left\|(t R+(1-t)(T \circ S))\left(\mathbf{x}_{n}\right)\right\| \rightarrow 1$ as $n \rightarrow \infty$, and hence $\|t R+(1-t)(T \circ S)\|=1$ and $\left(\mathbf{x}_{n}\right)_{n \in \mathbb{N}}$ is a supporting sequence for $t R+$ $(1-t)(T \circ S)$.

Corollary 3.5 Let X be a Hilbert space of dimension strictly greater than 3. Then $\mathcal{H B}(\mathrm{lim})$ is a nonmaximal face $\mathrm{B}_{\mathcal{L}_{0}^{X}}$.

Proof It suffices to notice that $X$ enjoys the Bade property and has a surjective linear isometry $T: X \rightarrow X$ with a nonzero fixed point (and thus a self-supporting sequence). Now we apply Theorem 3.1 and Theorem 3.4.

\subsection{The space $c_{\mathcal{C}}(X)$}

We will study in this subsection the space of convergent sequences through a set of operators in $\mathcal{H} \mathcal{B}(\mathrm{lim})$.

Definition 3.6 (Convergence method) Let $X$ be a normed space. Let $\mathcal{C}$ be a subset of $\mathcal{H B}(\lim )$. A sequence $\left(x_{n}\right)_{n \in \mathbb{N}} \in \ell_{\infty}(X)$ is said to be $\mathcal{C}$-convergent to $x \in X$ provided that $T\left(\left(x_{n}\right)_{n \in \mathbb{N}}\right)=x$ for all $T \in \mathcal{C}$, where $x$ is called the $\mathcal{C}$-limit of $\left(x_{n}\right)_{n \in \mathbb{N}}$ and is denoted by $\mathcal{C} \lim _{n \rightarrow \infty} x_{n}$. The space

$$
c_{\mathcal{C}}(X):=\left\{\left(x_{n}\right)_{n \in \mathbb{N}} \in \ell_{\infty}(X):\left(x_{n}\right)_{n \in \mathbb{N}} \text { is } \mathcal{C} \text {-convergent }\right\}
$$

is called the space of $\mathcal{C}$-convergent sequences and the map

$$
\begin{aligned}
\mathcal{C} \lim : & c_{\mathcal{C}}(X) \rightarrow X, \\
& \left(x_{n}\right)_{n \in \mathbb{N}} \mapsto \mathcal{C} \lim _{n \rightarrow \infty} x_{n}
\end{aligned}
$$

is called the $\mathcal{C}$-limit operator.

Example 3.7 (Banach limits) It was shown in [7, p. 316] that $\mathscr{B} \mathscr{L}\left(c_{0}\right)=\varnothing$ and in $[7$, Corollary 2] that $\operatorname{ac}(X) \subseteq c_{\mathscr{B} \mathscr{L}(X)}(X)$, where $\operatorname{ac}(X)$ is the space of almost convergent $X$-valued sequences for $X$ a normed space. In [18] Lorentz proved that ac $(\mathbb{R})=c_{\mathscr{B} \mathscr{L}(\mathbb{R})}(\mathbb{R})$. In [23] it was shown that $\mathscr{B} \mathscr{L}(\mathcal{B}(H)) \neq \varnothing$ and $\operatorname{ac}(\mathcal{B}(H)) \subsetneq c_{\mathscr{B} \mathscr{L}(\mathcal{B}(H))}(\mathcal{B}(H))$ for $H$ an infinite dimensional complex Hilbert space.

Theorem 3.8 Let $X$ be a normed space. Let $\mathcal{C}$ be a subset of $\mathcal{H B}(\mathrm{lim})$. Then:

(1) $c_{\mathcal{C}}(X)=\bigcup\left\{Z \subseteq \ell_{\infty}(X): T,\left.S \in \mathcal{C} \Rightarrow T\right|_{Z}=\left.S\right|_{Z}\right\}=\bigcap\{\operatorname{ker}(T-S): T, S \in \mathcal{C}\}=$ $\bigcap\left\{\operatorname{ker}\left(T_{0}-S\right): S \in \mathcal{C}\right\}$ for each $T_{0} \in \mathcal{C}$. Thus $c_{\mathcal{C}}(X)$ is a closed subspace of $\ell_{\infty}(X)$.

(2) If $\operatorname{dim}(X)=1$ and there exists $T_{0} \in \mathcal{C}$ for which $\operatorname{span}\left\{T_{0}-S: S \in \mathcal{C}\right\}$ is finite dimensional, then $\operatorname{dim}\left(\operatorname{span}\left\{T_{0}-S: S \in \mathcal{C}\right\}\right)=\operatorname{codim}\left(c_{\mathcal{C}}(X)\right)$ in $\ell_{\infty}(X)$.

(3) If $\mathcal{C} \subseteq \mathcal{D} \subseteq \mathcal{H B}(\lim )$, then $c_{\mathcal{D}}(X) \subseteq c_{\mathcal{C}}(X)$ and $\left.\mathcal{C} \lim \right|_{\mathcal{D}_{\mathcal{D}}(X)}=\mathcal{D} \lim$.

(4) $c_{\mathcal{C}}(X)=c_{\overline{\mathrm{co}}^{w}(\mathcal{C})}(X)$ and $\overline{\mathrm{co}}^{w}(\mathcal{C}) \lim =\mathcal{C}$ lim, where $w$ stands for the pointwise convergence topology $\sigma\left(\mathcal{B}\left(\ell_{\infty}(X), X\right),\left\{\delta_{\left(x_{n}\right)_{n \in \mathbb{N}}}:\left(x_{n}\right)_{n \in \mathbb{N}} \in \ell_{\infty}(X)\right\}\right)$.

(5) $c(X) \subseteq c_{\mathcal{C}}(X)$.

(6) $\left.\mathcal{C} \lim \right|_{c(X)}=\lim$.

(7) $\|\mathcal{C} \lim \|=1$.

(8) $c_{\mathcal{C}}(X)=\ell_{\infty}(X)$ if and only if $\mathcal{C}$ is a singleton. 
(1) Let $\left(x_{n}\right)_{n \in \mathbb{N}} \in c_{\mathcal{C}}(X)$. If we take $Z:=\left\{\left(x_{n}\right)_{n \in \mathbb{N}}\right\}$, then $\left.T\right|_{Z}=\left.S\right|_{Z}$ for all $T, S \in \mathcal{C}$.

Therefore $\left(x_{n}\right)_{n \in \mathbb{N}} \in \bigcup\left\{Z \subseteq \ell_{\infty}(X): T,\left.S \in \mathcal{C} \Rightarrow T\right|_{Z}=\left.S\right|_{Z}\right\}$. Now let $\left(x_{n}\right)_{n \in \mathbb{N}} \in \bigcup\left\{Z \subseteq \ell_{\infty}(X): T,\left.S \in \mathcal{C} \Rightarrow T\right|_{Z}=\left.S\right|_{Z}\right\}$. There exists $Z \subseteq \ell_{\infty}(X)$ verifying that $\left.T\right|_{Z}=\left.S\right|_{Z}$ for all $T, S \in \mathcal{C}$ and $\left(x_{n}\right)_{n \in \mathbb{N}} \in Z$. Pick any $T, S \in \mathcal{C}$. Then $T\left(\left(x_{n}\right)_{n \in \mathbb{N}}\right)=S\left(\left(x_{n}\right)_{n \in \mathbb{N}}\right)$ so $\left(x_{n}\right)_{n \in \mathbb{N}} \in \operatorname{ker}(T-S)$. The arbitrariness of $T$ and $S$ allows us to conclude that $\left(x_{n}\right)_{n \in \mathbb{N}} \in \bigcap\{\operatorname{ker}(T-S): T, S \in \mathcal{C}\}$. It is trivial that $\bigcap\{\operatorname{ker}(T-S): T, S \in \mathcal{C}\} \subseteq \bigcap\left\{\operatorname{ker}\left(T_{0}-S\right): S \in \mathcal{C}\right\}$ for every $T_{0} \in \mathcal{C}$. Finally, fix arbitrary $T_{0} \in \mathcal{C}$, and let $\left(x_{n}\right)_{n \in \mathbb{N}} \in \bigcap\left\{\operatorname{ker}\left(T_{0}-S\right): S \in \mathcal{C}\right\}$. If $S \in \mathcal{C}$, then $S\left(\left(x_{n}\right)_{n \in \mathbb{N}}\right)=T_{0}\left(\left(x_{n}\right)_{n \in \mathbb{N}}\right)$ because by hypothesis $\left(x_{n}\right)_{n \in \mathbb{N}} \in \operatorname{ker}\left(S-T_{0}\right)$. The arbitrariness of $S$ implies that $\left(x_{n}\right)_{n \in \mathbb{N}} \in c_{\mathcal{C}}(X)$ and $\mathcal{C} \lim _{n \rightarrow \infty} x_{n}=T_{0}\left(\left(x_{n}\right)_{n \in \mathbb{N}}\right)$.

(2) Recall first that if $\left\{z_{1}^{*}, \ldots, z_{k}^{*}\right\}$ is a finite linearly independent subset of the dual of a vector space $Z$, then $\operatorname{codim}\left(\bigcap_{i=1}^{k} \operatorname{ker}\left(z_{i}^{*}\right)\right)=k$. By relying on this and on the first isomorphism theorem, if $\left\{z_{j}^{*}: j \in J\right\}$ is a linearly independent subset of $Z^{*}$ such that $\operatorname{codim}\left(\bigcap_{j \in J} \operatorname{ker}\left(z_{j}^{*}\right)\right)$ is finite, then $\operatorname{card}(J)=\operatorname{codim}\left(\bigcap_{j \in J} \operatorname{ker}\left(z_{j}^{*}\right)\right)$. However, it does not hold that $\operatorname{dim}(F)=\operatorname{codim}\left(\bigcap_{z^{*} \in F} \operatorname{ker}\left(z^{*}\right)\right)$ for every vector subspace $F$ of $Z^{*}$. Indeed, assume that $Z$ is an infinite dimensional normed space of countable dimension, and take $F:=Z^{*}$, which is a Banach space and thus it has uncountable dimension. Then $F$ separates points of $Z$ by virtue of the Hahn-Banach theorem, and thus $\bigcap_{z^{*} \in F} \operatorname{ker}\left(z^{*}\right)=\{0\}, \operatorname{so~} \operatorname{codim}\left(\bigcap_{z^{*} \in F} \operatorname{ker}\left(z^{*}\right)\right)=\operatorname{dim}(Z)<\operatorname{dim}\left(Z^{*}\right)=\operatorname{dim}(F)$. Now, if $\operatorname{dim}\left(\operatorname{span}\left\{T_{0}-S: T \in \mathcal{C}\right\}\right)=n$, then there exist $S_{i} \in \mathcal{C}$ for $i=1, \ldots, n$ such that $\left\{T_{0}-S_{i}: i \in\{1, \ldots, n\}\right\}$ is a linear basis. Then $c_{\mathcal{C}}(X)=\bigcap_{S \in \mathcal{C}} \operatorname{ker}\left(T_{0}-S\right)=\bigcap_{i=1}^{n} \operatorname{ker}\left(T_{0}-S_{i}\right)$ has codimension $n$ in $\ell_{\infty}(X)$.

(3) If $\left(x_{n}\right)_{n \in \mathbb{N}} \in \mathcal{C}_{\mathcal{D}}(X)$, then there exists $x \in X$ such that $T\left(\left(x_{n}\right)_{n \in \mathbb{N}}\right)=x$ for all $T \in \mathcal{D}$, in particular the previous equality also holds for all $T \in \mathcal{C}$, therefore $\left(x_{n}\right)_{n \in \mathbb{N}} \in c_{\mathcal{C}}(X)$ and $x=\mathcal{C} \lim _{n \rightarrow \infty} x_{n}$.

(4) By (2), $c_{\mathcal{C}}(X) \supseteq c_{\overline{\mathrm{co}}^{w}(\mathcal{C})}(X)$. Let $\left(x_{n}\right)_{n \in \mathbb{N}} \in c_{\mathcal{C}}(X)$ and $T \in \overline{\mathrm{co}}^{w}(\mathcal{C})$. There exists a net $\left(T_{i}\right)_{i \in I} \subseteq \operatorname{co}(\mathcal{C})$ which is pointwise convergent to $T$. For every $i \in I$, we can write $T_{i}=\lambda_{1} T_{i_{1}}+\cdots+\lambda_{k_{i}} T_{i_{k_{i}}}$, where $T_{i_{j}} \in \mathcal{C}$ and $\lambda_{j} \geq 0$ for all $j \in\left\{1, \ldots, k_{i}\right\}$ and $\lambda_{1}+\cdots+\lambda_{k_{i}}=1$. Then

$$
\begin{aligned}
T\left(\left(x_{n}\right)_{n \in \mathbb{N}}\right) & =\lim _{i \in I} T_{i}\left(\left(x_{n}\right)_{n \in \mathbb{N}}\right) \\
& =\lim _{i \in I} \sum_{j=1}^{k_{i}} \lambda_{j} T_{i_{j}}\left(\left(x_{n}\right)_{n \in \mathbb{N}}\right) \\
& =\lim _{i \in I} \sum_{j=1}^{k_{i}} \lambda_{j} \mathcal{C} \lim _{n \rightarrow \infty} x_{n} \\
& =\lim _{i \in I} \mathcal{C} \lim _{n \rightarrow \infty} x_{n} \\
& =\mathcal{C} \lim _{n \rightarrow \infty} x_{n} .
\end{aligned}
$$

This shows that $\left(x_{n}\right)_{n \in \mathbb{N}} \in c_{\overline{\mathrm{co}}^{w}(\mathcal{C})}(X)$ and

$$
\overline{\mathrm{co}}^{w}(\mathcal{C}) \lim _{n \rightarrow \infty} x_{n}=\mathcal{C} \lim _{n \rightarrow \infty} x_{n}
$$


(5) Let $\left(x_{n}\right)_{n \in \mathbb{N}} \in c(X)$. For every $T \in \mathcal{C}$, since $\left.T\right|_{c(X)}=\lim$, we have that $T\left(\left(x_{n}\right)_{n \in \mathbb{N}}\right)=\lim _{n \rightarrow \infty} x_{n}$. This shows that $\left(x_{n}\right)_{n \in \mathbb{N}} \in c_{\mathcal{C}}(X)$.

(6) Let $\left(x_{n}\right)_{n \in \mathbb{N}} \in c(X)$. Fix any $T \in \mathcal{C}$. Then

$$
\mathcal{C} \lim _{n \rightarrow \infty} x_{n}=T\left(\left(x_{n}\right)_{n \in \mathbb{N}}\right)=\lim _{n \rightarrow \infty} x_{n}
$$

since $\left.T\right|_{c(X)}=\lim$.

(7) Since $\left.\mathcal{C} \lim \right|_{c(X)}=\lim$, we have that

$$
\|\mathcal{C} \lim \| \geq\left\|\left.\mathcal{C} \lim \right|_{c(X)}\right\|=\|\lim \|=1
$$

Now fix any $T \in \mathcal{C}$ and any $\left(x_{n}\right)_{n \in \mathbb{N}} \in \mathrm{B}_{c_{\mathcal{C}}(X)}$. Then

$$
\left\|\mathcal{C} \lim _{n \rightarrow \infty} x_{n}\right\|=\left\|T\left(\left(x_{n}\right)_{n \in \mathbb{N}}\right)\right\| \leq\|T\|\left\|\left(x_{n}\right)_{n \in \mathbb{N}}\right\| \leq 1
$$

(8) If there exists $T \in \mathcal{H B}(\lim )$ such that $\mathcal{C}=\{T\}$, then by (1) we have that

$$
c_{\mathcal{C}}(X)=\bigcup\left\{Z \subseteq \ell_{\infty}(X):\left.T\right|_{Z}=\left.T\right|_{Z}\right\}=\ell_{\infty}(X)
$$

Conversely, suppose that there are $T, S \in \mathcal{C}_{\mathcal{C}}(X)$. Then, by using (1) again, we have that $\ell_{\infty}(X)=c_{\mathcal{C}}(X) \subseteq \operatorname{ker}(T-S)$, which implies that $T=S$.

\section{$3.3 c(X)$ as $c_{\mathcal{C}}(X)$}

Recall that if $X$ is a normed space, then we let $\bar{X}$ denote its completion and if $x \in X$, then $\mathbf{x}$ stands for the constant sequence of general term $x$.

Theorem 3.9 Let $X$ be a normed space. Let $\mathcal{C}$ be a subset of $\mathcal{H B}(\mathrm{lim})$. Then $X$ is complete if and only if so is $c_{\mathcal{C}}(X)$.

Proof If $X$ is complete, then so is $\ell_{\infty}(X)$ and hence so is $c_{\mathcal{C}}(X)$ because it is closed in $\ell_{\infty}(X)$ in view of Theorem 3.8(1). Conversely, suppose that $c_{\mathcal{C}}(X)$ is complete. Let $\left(x_{n}\right)_{n \in \mathbb{N}}$ be a Cauchy sequence in $X$. Let $y \in \bar{X}$ be the limit of $\left(x_{n}\right)_{n \in \mathbb{N}}$. Note that $\left(\mathbf{x}_{\mathbf{n}}\right)_{n \in \mathbb{N}}$ is a Cauchy sequence in $c_{\mathcal{C}}(X)$, so there exists a sequence $z:=\left(z_{n}\right)_{n \in \mathbb{N}} \in c_{\mathcal{C}}(X)$ such that $\left(\mathbf{x}_{\mathbf{n}}\right)_{n \in \mathbb{N}}$ converges to $\left(z_{n}\right)_{n \in \mathbb{N}}$. Since $c_{\mathcal{C}}(X) \subseteq c_{\mathcal{C}}(\bar{X})$ and $\left(\mathbf{x}_{\mathbf{n}}\right)_{n \in \mathbb{N}}$ converges to both $\mathbf{y}$ and $z$ in $c_{\mathcal{C}}(\bar{X})$, we obtain that $z=\mathbf{y}$, and thus $z_{n}=y$ for all $n \in \mathbb{N}$ concluding that $y \in X$.

Our next results show that if $X$ is a noncomplete normed space, then there does not exist $\mathcal{C} \subseteq \mathcal{H B}(\lim )$ for which $c(X)=c_{\mathcal{C}}(X)$.

Lemma $3.10 c(X)$ is dense in $c(\bar{X})$.

Proof Let $\left(y_{n}\right)_{n \in \mathbb{N}} \in c(\bar{X})$ and fix arbitrary $\varepsilon>0$. For every $n \in \mathbb{N}$ let $x_{n} \in X$ such that $\| y_{n}-$ $x_{n} \| \leq \varepsilon / 2$. Take $n_{0} \in \mathbb{N}$ such that $1 / n_{0}<\varepsilon / 2$ and $\left\|y_{p}-y_{q}\right\|<\varepsilon / 2$ for all $p, q \geq n_{0}$. Then $\left(x_{1}, x_{2}, \ldots, x_{n_{0}-1}, x_{n_{0}}, x_{n_{0}}, x_{n_{0}}, \ldots\right) \in c(X)$ and

$$
\left\|\left(x_{1}, x_{2}, \ldots, x_{n_{0}-1}, x_{n_{0}}, x_{n_{0}}, x_{n_{0}}, \ldots\right)-\left(y_{n}\right)_{n \in \mathbb{N}}\right\|_{\infty}<\varepsilon
$$


since

$$
\left\|x_{n_{0}}-y_{n}\right\| \leq\left\|x_{n_{0}}-y_{n_{0}}\right\|+\left\|y_{n_{0}}-y_{n}\right\|<\frac{\varepsilon}{2}+\frac{\varepsilon}{2}=\varepsilon
$$

for all $n \geq n_{0}$.

It is easy to check that $c(X)$ is never dense in $\ell_{\infty}(X)$. When $X=\mathbb{R}$, a separability argument can be applied. In general, one can see that a sequence like $\left((-1)^{n} x\right)_{n \in \mathbb{N}}$ can never be approximated by convergent sequences in the sup norm if $x \neq 0$.

Theorem 3.11 The following conditions are equivalent for a normed space $X$ :

(1) $c(\bar{X}) \cap \ell_{\infty}(X)=c(X)$

(2) $c(X)$ is a closed subspace of $\ell_{\infty}(X)$.

(3) $X$ is complete.

\section{Proof}

$1 \Rightarrow 2$ Immediate if taken into account that $c(\bar{X})$ is closed in $\ell_{\infty}(\bar{X})$ since $\bar{X}$ is complete.

$2 \Rightarrow 3$ Note that $c(\bar{X}) \cap \ell_{\infty}(X)$ is a closed subspace of $\ell_{\infty}(X)$ containing $c(X)$. In view of Lemma 3.10, we have that $c(X)$ is dense in $c(\bar{X})$, therefore $c(\bar{X}) \cap \ell_{\infty}(X)=c(X)$ by hypothesis. Consider now a Cauchy sequence $\left(x_{n}\right)_{n \in \mathbb{N}} \subset X$. It is obvious that $\left(x_{n}\right)_{n \in \mathbb{N}} \in c(\bar{X}) \cap \ell_{\infty}(X)=c(X)$. So $\left(x_{n}\right)_{n \in \mathbb{N}}$ is convergent in $X$.

$3 \Rightarrow 1$ Obvious since $X=\bar{X}$.

Corollary 3.12 Let $X$ be a noncomplete normed space. There does not exist $\mathcal{C} \subseteq \mathcal{H B}(\mathrm{lim})$ for which $c(X)=c_{\mathcal{C}}(X)$.

Proof Assume to the contrary that there exists $\mathcal{C} \subseteq \mathcal{H} \mathcal{B}(\lim )$ for which $c(X)=c_{\mathcal{C}}(X)$. In view of Theorem 3.8(1), we have that $c(X)$ is a closed subspace of $\ell_{\infty}(X)$. By applying Theorem 3.11, we obtain that $X$ is complete.

Lemma 3.13 Let $X$ be a normed space. Let $\left(x_{n}\right)_{n \in \mathbb{N}} \subseteq X$ be a convergent sequence to $x \in X$ and $\left(a_{n}\right)_{n \in \mathbb{N}} \subseteq X$ be a nonconvergent bounded sequence with a w-convergent subnet $\left(a_{n_{i}}\right)_{i \in I}$ to $a \in X$. Then

$$
\|x+\lambda a\| \leq \sup _{n \in \mathbb{N}}\left\|x_{n}+\lambda a_{n}\right\|
$$

for all $\lambda \in \mathbb{K}$. As a consequence, the operator

$$
\begin{aligned}
& c(X) \oplus \mathbb{K}\left(a_{n}\right)_{n \in \mathbb{N}} \rightarrow \mathbb{K}, \\
& \left(y_{n}\right)_{n \in \mathbb{N}}+\lambda\left(a_{n}\right)_{n \in \mathbb{N}} \mapsto \lim _{n \rightarrow \infty} y_{n}+\lambda a
\end{aligned}
$$

has norm 1 . Also, $\left(a_{n}\right)_{n \in \mathbb{N}}$ cannot be approximated by $c(X)$.

Proof The net $\left(x_{n_{i}}+\lambda a_{n_{i}}\right)_{i \in I} w$-converges to $x+\lambda a$. The $w$-lower semicontinuity of the norm implies that

$$
\|x+\lambda a\| \leq \liminf _{i \in I}\left\|x_{n_{i}}+\lambda a_{n_{i}}\right\| \leq \sup _{i \in I}\left\|x_{n_{i}}+\lambda a_{n_{i}}\right\| \leq \sup _{n \in \mathbb{N}}\left\|x_{n}+\lambda a_{n}\right\| .
$$


This shows that map (3.1) has norm less than or equal to 1 . The fact that map (3.1) is an extension of the limit function forces it to have norm 1. Finally, suppose that $\left(a_{n}\right)_{n \in \mathbb{N}}$ is approximated by $c(X)$. Then $\left(a_{n}\right)_{n \in \mathbb{N}}$ is convergent in $\bar{X}$. As a consequence, $\left(a_{n}\right)_{n \in \mathbb{N}}$ is convergent in $\bar{X}$ to $a \in X$, which means that $\left(a_{n}\right)_{n \in \mathbb{N}}$ is convergent in $X$ to $a$.

Theorem 3.14 Let $X$ be a reflexive 1-injective Banach space. Then $c(X)=c_{\mathcal{H}(\mathrm{Blim})}(X)$.

Proof Suppose to the contrary that there exists $\left(a_{n}\right)_{n \in \mathbb{N}} \in c_{\mathcal{H}(\mathrm{B}(\mathrm{lim})}(X) \backslash c(X)$. Since $X$ is finite dimensional, there are two subsequences $\left(a_{n_{k}}\right)_{k \in \mathbb{N}}$ and $\left(a_{m_{k}}\right)_{k \in \mathbb{N}}$ convergent to different elements $a$ and $b$, respectively. Now consider the maps

$$
\begin{aligned}
S_{a}: \quad c(X) \oplus \mathbb{K}\left(a_{n}\right)_{n \in \mathbb{N}} \rightarrow \mathbb{K}, \\
\left(x_{n}\right)_{n \in \mathbb{N}}+\lambda\left(a_{n}\right)_{n \in \mathbb{N}} \mapsto \lim _{n \rightarrow \infty} x_{n}+\lambda a
\end{aligned}
$$

and

$$
\begin{aligned}
S_{b}: \quad c(X) \oplus \mathbb{K}\left(a_{n}\right)_{n \in \mathbb{N}}, \rightarrow \mathbb{K}, \\
\quad\left(x_{n}\right)_{n \in \mathbb{N}}+\lambda\left(a_{n}\right)_{n \in \mathbb{N}} \mapsto \lim _{n \rightarrow \infty} x_{n}+\lambda b .
\end{aligned}
$$

According to Lemma 3.13, $\left\|S_{a}\right\|=\left\|S_{b}\right\|=1$. Also, $\left.S_{a}\right|_{c(X)}=\left.S_{b}\right|_{c(X)}=\lim$. Since $X$ is 1injective, there are $T_{a}, T_{b} \in \mathcal{H B}(\mathrm{lim})$ such that $\left.T_{a}\right|_{c(X) \oplus \mathbb{K}\left(a_{n}\right)_{n \in \mathbb{N}}}=S_{a}$ and $\left.T_{b}\right|_{c(X) \oplus \mathbb{K}\left(a_{n}\right)_{n \in \mathbb{N}}}=$ $S_{b}$. Now, by hypothesis, we obtain the contradiction that

$$
a=S_{a}\left(\left(a_{n}\right)_{n \in \mathbb{N}}\right)=T_{a}\left(\left(a_{n}\right)_{n \in \mathbb{N}}\right)=T_{b}\left(\left(a_{n}\right)_{n \in \mathbb{N}}\right)=S_{b}\left(\left(a_{n}\right)_{n \in \mathbb{N}}\right)=b .
$$

\subsection{Multipliers}

This final subsection serves to define multipliers for the convergence through operators.

Definition 3.15 Let $X$ and $Y$ be normed spaces. Consider $\mathcal{D} \subseteq \mathcal{H} \mathcal{B}_{Y}(\lim )$. Then:

(1) The $\mathcal{D}$-multiplier space associated with a sequence $\left(T_{n}\right)_{n \in \mathbb{N}} \subseteq \mathcal{B}(X, Y)$ is defined as

$$
\mathcal{M}_{\mathcal{D}}^{\infty}\left(\left(T_{n}\right)_{n \in \mathbb{N}}\right):=\left\{\left(x_{n}\right)_{n \in \mathbb{N}} \in \ell_{\infty}(X): \sum_{n=1}^{\infty} T_{n}\left(x_{n}\right) \in c_{\mathcal{D}}(Y)\right\}
$$

and the $\mathcal{D}$-summing operator associated with $\left(T_{n}\right)_{n \in \mathbb{N}}$ is defined as

$$
\begin{aligned}
\mathcal{D} \sum_{n=1}^{\infty} T_{n}: & \mathcal{M}_{\mathcal{D}}^{\infty}\left(\left(T_{n}\right)_{n \in \mathbb{N}}\right) \rightarrow Y, \\
& \left(x_{n}\right)_{n \in \mathbb{N}} \mapsto \mathcal{D} \sum_{n=1}^{\infty} T_{n}\left(x_{n}\right) .
\end{aligned}
$$

(2) The $\mathcal{D}$-summability space associated with a subspace $\mathcal{S} \subseteq \ell_{\infty}(X)$ is defined as

$$
\mathcal{B}(X, Y)(\mathcal{S}):=\left\{\left(T_{n}\right)_{n \in \mathbb{N}} \in \mathcal{B}(X, Y)^{\mathbb{N}}: \mathcal{S} \subseteq \mathcal{M}_{\mathcal{D}}^{\infty}\left(\left(T_{n}\right)_{n \in \mathbb{N}}\right)\right\}
$$


Recall that in [7, Corollary 2] it was proved that $\operatorname{ac}(X) \subseteq c_{\mathscr{B} \mathscr{L}(X)}(X)$. This result allows us to conclude the following.

Proposition 3.16 Let $X$ and $Y$ be normed spaces and $\left(T_{n}\right)_{n \in \mathbb{N}} \subseteq \mathcal{B}(X, Y)$. Take $\left.\mathscr{C}\right|_{\ell_{\infty}(Y)}$ : $\ell_{\infty}(Y) \rightarrow \ell_{\infty}\left(\ell_{\infty}(Y)\right)$ the general Cesáro operator restricted to $\ell_{\infty}(Y)$. Then

$$
\mathcal{M}_{\mathscr{B} \mathscr{L}(Y)}^{\infty}\left(\left(T_{n}\right)_{n \in \mathbb{N}}\right) \supseteq \mathcal{M}_{\left.\mathscr{C}\right|_{\ell_{\infty}(Y)}}^{\infty}\left(\left(T_{n}\right)_{n \in \mathbb{N}}\right)
$$

Proof Simply observe that by combining [7, Corollary 2] with Theorem 2.15, we obtain

$$
\begin{aligned}
\mathcal{M}_{\mathscr{B} \mathscr{L}(Y)}^{\infty}\left(\left(T_{n}\right)_{n \in \mathbb{N}}\right) & =\left\{\left(x_{n}\right)_{n \in \mathbb{N}} \in \ell_{\infty}(X): \sum_{n=1}^{\infty} T_{n}\left(x_{n}\right) \in c_{\mathscr{B} \mathscr{L}(Y)}(Y)\right\} \\
& \supseteq\left\{\left(x_{n}\right)_{n \in \mathbb{N}} \in \ell_{\infty}(X): \sum_{n=1}^{\infty} T_{n}\left(x_{n}\right) \in \operatorname{ac}(Y)\right\} \\
& \supseteq\left\{\left(x_{n}\right)_{n \in \mathbb{N}} \in \ell_{\infty}(X): \sum_{n=1}^{\infty} T_{n}\left(x_{n}\right) \in c_{\left.\mathscr{C}\right|_{\ell_{\infty}(Y)}}(Y)\right\} \\
& =\mathcal{M}_{\left.\mathscr{C}\right|_{\ell_{\infty}(Y)}}\left(\left(T_{n}\right)_{n \in \mathbb{N}}\right) .
\end{aligned}
$$

\section{Acknowledgements}

The first and third authors are supported by Ministerio de Ciencia, Innovación y Universidades under

PGC2018-101514-B-100, by Junta de Andalucía FQM-257, and Plan Propio de la Universidad de Cádiz. This work has been co-financed by the 2014-2020 ERDF Operational Programme and by the Department of Economy, Knowledge, Business and University of the Regional Government of Andalusia. Project reference: FEDER-UCA18-108415.

\section{Funding}

The first and third authors are supported by Ministerio de Ciencia, Innovación y Universidades under

PGC2018-101514-B-100, by Junta de Andalucía FQM-257, and Plan Propio de la Universidad de Cádiz. The third author is supported by the 2014-2020 ERDF Operational Programme and by the Department of Economy, Knowledge, Business and University of the Regional Government of Andalusia. Project reference: FEDER-UCA18-108415.

\section{Availability of data and materials}

Not applicable.

\section{Competing interests}

The authors declare that they have no competing interests.

\section{Authors' contributions}

The authors contributed equally and significantly in writing this paper. All the authors read and approved the final manuscript.

\section{Author details}

${ }^{1}$ Department of Mathematics, University of Cadiz, Puerto Real 11510, Spain. ${ }^{2}$ Department of Mathematics and Physical Sciences Education, Faculty of Education, Siirt University, The Kezer Campus, Kezer, 56100 Siirt, Turkey.

\section{Publisher's Note}

Springer Nature remains neutral with regard to jurisdictional claims in published maps and institutional affiliations.

Received: 8 June 2020 Accepted: 17 March 2021 Published online: 01 April 2021

\section{References}

1. Aizpuru, A., Armario, R., García-Pacheco, F.., Pérez-Fernández, F.J.: Banach limits and uniform almost summability. J. Math. Anal. Appl. 379(1), 82-90 (2011)

2. Aizpuru, A., Armario, R., García-Pacheco, F.., Pérez-Fernández, F.J.: Vector-valued almost convergence and classical properties in normed spaces. Proc. Indian Acad. Sci. Math. Sci. 124(1), 93-108 (2014)

3. Aizpuru, A., García-Pacheco, F.J.: A short note about exposed points in real Banach spaces. Acta Math. Sci. Ser. B Engl. Ed. 28(4), 797-800 (2008)

4. Aizpuru, A., Listán-García, M.C., Rambla-Barreno, F.: Double density by moduli and statistical convergence. Bull. Belg. Math. Soc. Simon Stevin 19(4), 663-673 (2012) 
5. Aizpuru, A., Listán-García, M.C., Rambla-Barreno, F.: Density by moduli and statistical convergence. Quaest. Math. 37(4), 525-530 (2014)

6. Altay, B., Kama, R.: On Cesàro summability of vector valued multiplier spaces and operator valued series. Positivity 22(2), 575-586 (2018)

7. Armario, R., Garcsiya-Pacheko, F.Kh., Peres-Fernandes, F.Kh.: On vector-valued Banach limits. Funkc. Anal. Prilozh. 47(4), 82-86 (2013)

8. Bruck, R.E., Reich, S.: Accretive operators, Banach limits, and dual ergodic theorems. Bull. Acad. Polon. Sci. 29, 585-589 (1981)

9. Day, M.M.: Normed Linear Spaces. Springer, Berlin (1973)

10. Diestel, J.: Geometry of Banach Spaces-Selected Topics. Springer, Berlin (1975)

11. Fast, H.: Sur la convergence statistique. Colloq. Math. 2, 241-244 (1951)

12. García-Pacheco, F.J.: Convex components and multi-slices in real topological vector spaces. Ann. Funct. Anal. 6(3), 73-86 (2015)

13. García-Pacheco, F..: Extremal properties of the set of vector-valued Banach limits. Open Math. 13(1), 757-767 (2015)

14. Garsiya-Pacheko, F.Kh., Peres-Fernandes, F.Kh.: Fundamental aspects of vector-valued Banach limits. Izv. Ross. Akad. Nauk, Ser. Mat. 80(2), 33-46 (2016)

15. Kama, R.: On some vector valued multiplier spaces with statistical Cesàro summability. Filomat 33(16), 5135-5147 (2019)

16. Karakuş, M., Başar, F:: Operator valued series, almost summability of vector valued multipliers and (weak) compactness of summing operator. J. Math. Anal. Appl. 484(1), 123651, 16 pp. (2020)

17. Kelley, J.L.: Banach spaces with the extension property. Trans. Am. Math. Soc. 72, 323-326 (1952)

18. Lorentz, G.G.: A contribution to the theory of divergent sequences. Acta Math. 80, 167-190 (1948)

19. Reich, S.: Almost convergence and nonlinear ergodic theorems. J. Approx. Theory 4, 269-272 (1978)

20. Steinhaus, H.: Sur la convergence ordinaire et la convergence asymptotique. Colloq. Math. 2, 73-74 (1951)

21. Swartz, C.: Multiplier Convergent Series. World Scientific, Singapore (2009)

22. Swartz, C.: Operator valued series and vector valued multiplier spaces. Casp. J. Math. Sci. 3(2), 277-288 (2014)

23. Tanaka, R.: On vector-valued Banach limits with values in $\mathcal{B}(\mathcal{H})$. Publ. Math. (Debr.) $92(3-4)$, 471-480 (2018)

\section{Submit your manuscript to a SpringerOpen ${ }^{\circ}$ journal and benefit from:}

- Convenient online submission

- Rigorous peer review

- Open access: articles freely available online

- High visibility within the field

- Retaining the copyright to your article

Submit your next manuscript at $\gg$ springeropen.com 